\title{
Osteoarthritis in Italy: Impact on Health-Related Quality of Life and Health Care Resources
}

Patrizia Berto ${ }^{1}$, Maria Domenica Sanna ${ }^{1}$, James Jackson ${ }^{2}$, Mia Berry ${ }^{2}$, Jessica Jackson ${ }^{2}$

Regulatory Pharma Net srl, Pisa, Italy

Adelphi Real World, Bollington, UK

\begin{abstract}
OBJECTIVE: To determine how osteoarthritis (OA) severity correlates with self-reported outcomes relevant from the patient's perspective in the Italian clinical setting.

METHODS: Data were drawn from the 2017-18 Adelphi OA Disease Specific ProgrammeTM (DSP). Data were collected in the Italian clinical practice settings by primary care physicians, rheumatologists, orthopedists, and their patients with $\mathrm{OA}$, during their regular office visits. Physicians completed information about OA-related visits to healthcare professionals (HCPs), tests/scans conducted, emergency room (ER) visits, surgeries, and OA-related treatment. Physicians also rated patients' functioning on a 0 to 10 scale $(0=$ fully functional; $10=$ completely impaired). Outcomes included Western Ontario and McMaster Universities Osteoarthritis Index (WOMAC) score, functional limitations, treatment needs, EuroQoL-5 Dimension (EQ-5D) visual analogue scale (VAS), the work productivity and activity impairment. Descriptive statistics (numbers and percent for categorical variables; means with standard deviations [SD] for continuous variables) were used to evaluate the different variables as appropriate.

RESULTS: The study population comprised 900 patients from Italy with knee (40.9\%), back (38.7\%), hip (27.9\%), and/ or shoulder $(20.3 \%)$ OA. Mean age was 66.6 years with a prevalence of female $(63 \%)$ patients. Patients had mild $(26 \%)$, moderate (54\%), severe (20\%) disease severity. Patients with severe disease reported higher functional limitations, greater use of treatments, reduced quality of life, and impaired work productivity and activity. The burdens were higher among elderly and obese patients and in patients with highest pain severity score.

CONCLUSIONS: The results from this cross-sectional study show the impact of OA disease severity on all dimensions of health-related quality of life (HRQoL), as well as in OA-related health care resource use.
\end{abstract}

\section{Keywords}

Daily activity; Health-related quality of life; Osteoarthritis; Pain; Physical functioning; Work productivity

\section{INTRODUCTION}

Osteoarthritis (OA) is a progressive chronic disease potentially affecting every articular tissue and may potentially lead to joint failure. It has heterogeneous manifestation and, does not always have the same appearance in all patients. Indeed, OA may present differently in men and women, in patients with or without trauma, in athletes, or in obese patients [1,2]. OA articular cartilage changes result from the disruption of the normal balance between anabolic and catabolic processes, as well as cellular abnormalities in autocrine, paracrine, and endocrine regulation, leading to a disequilibrium in normal tissue turnover within the joint [2].

Numerous factors have been associated with an increased risk for the development of OA. These include systemic factors such as genetics, age and gender, sex hormones, bone mineral density, as well as local mechanical factors such as joint overload caused by obesity, acute injury, repetitive joint loading, and joint deformity [3]. Despite the multifactorial nature of $\mathrm{OA}$, the pathological changes seen in osteoarthritic joints have common features that affect the entire joint structure resulting in pain, deformity, and loss of function [4].

OA can be defined in terms of radiologic changes as well as symptoms, although there may be a discordance between these findings since many people with pathologic and radiographic evidence of osteoarthritis have no symptoms [5]. From a clinical perspective, pain is the disease characteristic that negatively affects multiple aspects of patient's life, including mobility, sleep, mood, and health related quality of life (HRQoL) [6]. Pain and function

Corresponding author Maria Domenica Sanna m.sanna@regulatorypharmanet.com

Received: 16 February 2021 Accepted: 6 April 2021 Published: 20 April 2021 
are core symptomatic outcomes of OA that are frequently targeted as part of pharmacologic therapy, and it may be expected that these outcomes are associated with patient perceptions of OA severity [7].

A variety of pharmacologic options are available for managing OA-related pain such as simple analgesics, nonsteroidal anti-inflammatory drugs (NSAIDs), oral corticosteroids, opioids, and injectables including corticosteroids and viscosupplementation with hyaluronan [8]. As occurs with most chronic conditions, adherence to OA medications is low. Factors implicated in adherence to OA include dosing frequency, self-efficacy levels, and physician and patient treatment satisfaction [9].

While simple analgesics and NSAIDs are generally recommended as first-line pharmacologic therapy, in clinical practice choosing among these medications is often determined by disease severity [10]. In clinical trials, definitions of severity are generally based on cut-points for patient-reported pain, function, and global assessments. However, in real-world settings, categorizing mild, moderate, or severe disease based solely on pain cut-points may be neither comprehensive nor relevant from the patient's perspective. Other factors such as functional impairment and work productivity may also contribute to a patient's overall perception of disease severity $[11,12]$. As a result, patients with OA report impairment of their ability to carry out activities of daily living in addition to deterioration in HRQoL.

As a consequence, OA patients are very high users of health care services and incur higher costs than patients without OA, due to the duration of disease and the level of impairment related to their illness [13]. The burden of OA is therefore considerable, both from a societal and individual patient perspective [14]. OA is responsible for a very high number of primary healthcare visits as well as knee and hip replacement procedures, plus hospital admission costs, as well as assistance for follow-up and rehabilitation [15]. However, the socio-economic burden of OA is not only limited to the direct costs of healthcare use, but also includes significant non-healthcare-related costs. These take the form of productivity losses and the cost of formal and informal care associated with the limited independence of people with osteoarthritis [15].

Considering Disability Adjusted Life Years (DALYs) over the period 1990-2010, OA burden of disease has grown at a faster pace than musculoskeletal diseases (64\% vs 45\%) [16]. According to the Italian Health Ministry, OA resulted to be the 7 th out of 283 diagnosis for Clinical Classifications Software (CCS) for number of hospital discharges from hospital data collected in 2014 [16]. Hospital discharges for OA in Italy were 127,000 , close to the $2 \%$ of the discharges with an average hospital stay of 7.5 days [17]. In Italy annual total costs per patient have been estimated $€ 3,000$ ( $€ 1,300$ as direct health-related costs and $€ 1,700$ as indirect costs adjusted in 2013) [18]. Recently, Berto and Aiello conducted a literature review, revealing that the impact of OA on the Health System, generates direct health costs related to hospital admissions for hip and knee surgery; and may generate additional costs due to relapses, need for intervention review, adverse events (e.g., infections, thrombosis and pulmonary embolisms) and subsequently to the rehabilitation process, in hospital and at home. They also reported the burden of illness also generated by indirect costs characterized by the reduced or lack of productivity of patient and family members, as patients with OA are affected by severe limitations in daily and work activities [19].

Overall, OA is a chronic condition that impacts significantly on life expectancy and patients' HRQoL and generates considerable costs to most health care systems. As with any chronic condition, non-adherence to the available pharmacological treatments is a problem that has the potential to impact on population health and expenditure.

Given that OA exerts multiple effects on patients' lives, the objective of this study was to determine how patient-rated OA severity (mild, moderate and severe) can affect self-reported outcomes relevant from the patient's perspective, including assistance with mobility and daily activities. Additionally, this study aims to understand the pattern of healthcare resource utilization (HCRU) in Italy as OA severity worsens.

\section{METHODS}

\section{Study Design}

This study utilized data from the 2017-18 Osteoarthritis Adelphi Disease Specific Programme (DSP) ${ }^{\mathrm{TM}}$. The Adelphi DSP is a large, multinational, point in time survey designed to capture a cross-section of real-world data for a range of common chronic diseases [20]. Data were collected in Italian clinical practice settings from primary care physicians, rheumatolo- 
gists, orthopedists, and their patients with OA, during their regular office visits. Physicians completed patient record forms (PRFs) on their next 9 consecutive patients $\geq 18$ years) consulting for OA, plus an oversample of 1 patient who had tried at least 3 prescribed medications for their OA pain. These patients were also invited to participate by providing written informed consent and completing a patient self-completion questionnaire (PSC). Physicians completed information about OA-related visits to healthcare professionals (HCPs), tests/scans conducted, emergency room (ER) visits, surgeries, and OA-related treatment. Physicians also rated patients' functioning on a 0 to 10 scale $(0=$ fully functional; $10=$ completely impaired $)$.

OA pain intensity and physical function were measured using the Western Ontario and McMaster Universities Osteoarthritis Index (WOMAC), a widely used, validated, self-administered, disease-specific questionnaire [21]. Patients rated their average pain intensity over the last week on a $0-10$ scale $(0=$ no pain; $10=$ worst possible pain $)$ and, using the pain component of the WOMAC, were categorized into mild (0-3), moderate (4-6) and severe (7-10) pain groups.

Patients also provided an assessment of their physical function (0-10 WOMAC scale where higher scores indicated greater functional impairment), impact on mobility, whether caregiver assistance was required, and daily activities requiring caregiver assistance.

The analysis was conducted using combined data from the total patient sample (random sample + over-sample). Not all physicians and patients answered all the questions on the PRF and PSC, respectively. Consequently, the respondent population size may differ for individual questions and thus for certain analyses. The population size is given for each individual analysis where appropriate and indicates the number of respondents (physician or patient) who provided responses relevant to that analysis.

\section{Outcome Measures}

Outcomes included measures for physical functioning, HRQoL, pain, work productivity and daily activity. Physical functioning was reported by patients using pain, physical function and stiffness scores from the WOMAC which are scored on a range from 0 to 10 . Generic HRQoL was assessed using the EuroQoL-5 Dimension (EQ-5D) instrument [22]. The EQ-5D includes a separate visual analogue scale (VAS) which varies from $0=$ worst health you can imagine to $100=$ best health you can imagine [22]. Work productivity and daily activity were assessed using the patient-reported Work Productivity and Activity Impairment Questionnaire (WPAI). WPAI scores are based on 1-item (presenteeism, activity impairment), 2-items (absenteeism) and multiple items (overall work productivity).

\section{Statistical Analysis}

Descriptive statistics (numbers and percent for categorical variables; means with standard deviations [SD] for continuous variables) were used to evaluate the different variables as appropriate.

\section{RESULTS}

\section{Demographic and Clinical Characteristics}

A total of 90 physicians were included in the analysis. Physicians' specialties were primary care (50), orthopedists (30) and rheumatologists (10). Each physician included 10 patients: 500 patients from primary care, 300 patients from orthopedists and 100 patients from rheumatologists providing data for a total of 900 patients. Physicians provided information from patient records on patient demographics and clinical characteristics including the random sample as well as the over-sample.

As shown in Table I, which presents the demographic characteristics, individuals were predominantly female $(63 \%)$ and the mean age was 66.6 years (SD 12.4). The mean body mass index (BMI) was 26.3 (SD 3.8), with $25 \%$ of patients being reported as obese (BMI $>30$ ) by physicians. There was a high presence $(46.5 \%)$ of patients who were retired, and about one third (32.1\%) were employed (25.9\% in full time, $6.2 \%$ in part-time employment). Only $3 \%$ of patients reported being retired due to OA.

Among the study sample, 237 (26\%) patients were classified by physicians as having mild OA, 482 (54\%) moderate OA and 181 (20\%) had severe OA (Table I). Among 900 patients, $699(129 \%)$ patients reported additional conditions on top of their OA. The most frequently were cardiovascular conditions (66\%), neurological conditions (33\%) and diabetes/endocrine diseases 32\% (Table I). 


\begin{tabular}{|c|c|}
\hline Characteristic & Value \\
\hline Mean age, years $(n=900)$ & 66.6 \\
\hline Gender, \% M/F (n=889) & $37 / 63$ \\
\hline BMI $(n=899)$ & 26.3 \\
\hline \multicolumn{2}{|c|}{ Employment Status, \% $(n=900)$} \\
\hline Retired & 46.5 \\
\hline Full time & 25.9 \\
\hline Homemaker & 19.5 \\
\hline Part time & 6.2 \\
\hline Unemployed & 1.1 \\
\hline On long term sick leave & 0.6 \\
\hline Student & 0.3 \\
\hline \multicolumn{2}{|c|}{ Disease severity, \% $(n=890)$} \\
\hline Mild & 26 \\
\hline Moderate & 20 \\
\hline Severe & 54 \\
\hline \multicolumn{2}{|c|}{ Concomitant medical conditions, $\%(n=699)$} \\
\hline Cardiovascular & 66 \\
\hline Diabetes/endocrine & 32 \\
\hline Neurological & 33 \\
\hline Asthma/COPD & 10 \\
\hline $\begin{array}{l}\text { Osteoporosis; back pain; } \\
\text { neuropathic pain; RA }\end{array}$ & 26 \\
\hline Any other & 25 \\
\hline \multicolumn{2}{|c|}{ Disease progression, $\%(n=900)$} \\
\hline Improving & 10 \\
\hline Stable & 45 \\
\hline Deteriorating slowly & 38 \\
\hline Deteriorating rapidly & 7 \\
\hline \multicolumn{2}{|l|}{ Joints affected, \% $(n=900)$} \\
\hline Knee & 40 \\
\hline Back & 38.7 \\
\hline Hip & 27.9 \\
\hline Shoulder & 20.3 \\
\hline Hand & 17.1 \\
\hline Neck & 15.7 \\
\hline Wrist & 8.3 \\
\hline Foot & 5.2 \\
\hline Elbow & 4.6 \\
\hline Thumb & 3.9 \\
\hline Ankle & 3.4 \\
\hline \multicolumn{2}{|l|}{ Functionality, \% ( $n=900)$} \\
\hline 0 - Fully functional & 6 \\
\hline 1 & 4 \\
\hline 2 & 8 \\
\hline 3 & 9 \\
\hline 4 & 12 \\
\hline 5 & 19 \\
\hline 6 & 16 \\
\hline 7 & 14 \\
\hline 8 & 10 \\
\hline 9 & 3 \\
\hline 10 - completely impaired & 0 \\
\hline
\end{tabular}

Table I. Demographics and clinical characteristics of patients with $O A$
As to the clinical profile $7 \%$ of patients were classified as rapidly deteriorating, $38 \%$ as slowly deteriorating, $45 \%$ were reported as having a stable disease and $10 \%$ as currently improving their disease status. Joints mostly affected by OA were the knee $(40.9 \%)$ and the back $(38.7 \%)$ followed by the hip $(27.9 \%)$ and the shoulder $(20.3 \%)$, with relatively lower frequencies for the hand, neck and wrist. Looking at patient's functionality, as recorded by the treating physician, patients appeared to stand on the higher area of the spectrum, with $43 \%$ of patients being rated 6 or higher (on a scale $0-10$ where 0 implies full functionality and 10 complete impairment) (Table I).

The average number of OA-related visits to the physician reported in the past 3 months by patients was 3.75 and an additional 1 visit was reported to a different health care professional (HCP) (on average, about 19/year). The average number of medical and other HCP visits appeared higher in the elderly (age group 74-89), in patients with higher pain severity (NRS 7-10) and in severe patients (according to both patients' and physicians' assessed disease severity) (Supplementary Figure 1). Severe patients reported respectively 24.3 and 22.3 visits per year (severity assessed by patients and physicians, respectively).

\section{Prescribed OA Treatment Patterns}

Exposure to pain-related treatments among OA patients was assessed in relation to disease progression, disease severity and pain severity.

Duration of treatment was the lowest in patients deteriorating rapidly; in (both rapidly and slowly) deteriorating patients on average 2 classes of drugs were used. The highest rate of strong opioids (46\%) and analgesics (38\%) use was recorded in patients deteriorating rapidly. In stable and improving patients, strong opioids were used only by a minority of patients $(7 \%$ and $5 \%$, respectively) (Figure 1).

No difference in duration of treatment by severity of disease was reported. Severe patients were prescribed 2 classes of drugs (vs 1 in mild and moderate patients); severe patients were more likely to be prescribed strong opioids $(37 \%)$, weak opioids $(26 \%)$ and analgesics (32\%), whilst moderate patients used more NSAIDs (74\%), but still one in five $(20 \%)$ of moderate patients used

weak opioids (Figure 2).

Duration of treatment by severity of pain (NRS 0-10), revealed no differences although there seemed to be a tendency towards a longer treatment duration with the decrease of pain severity. Severe pain patients (NRS 7-10) and moderate pain patients (NRS 4-6) used 2 classes of drugs (vs 1 in mild pain patients NRS 0-3); severe pain patients used more of all classes 


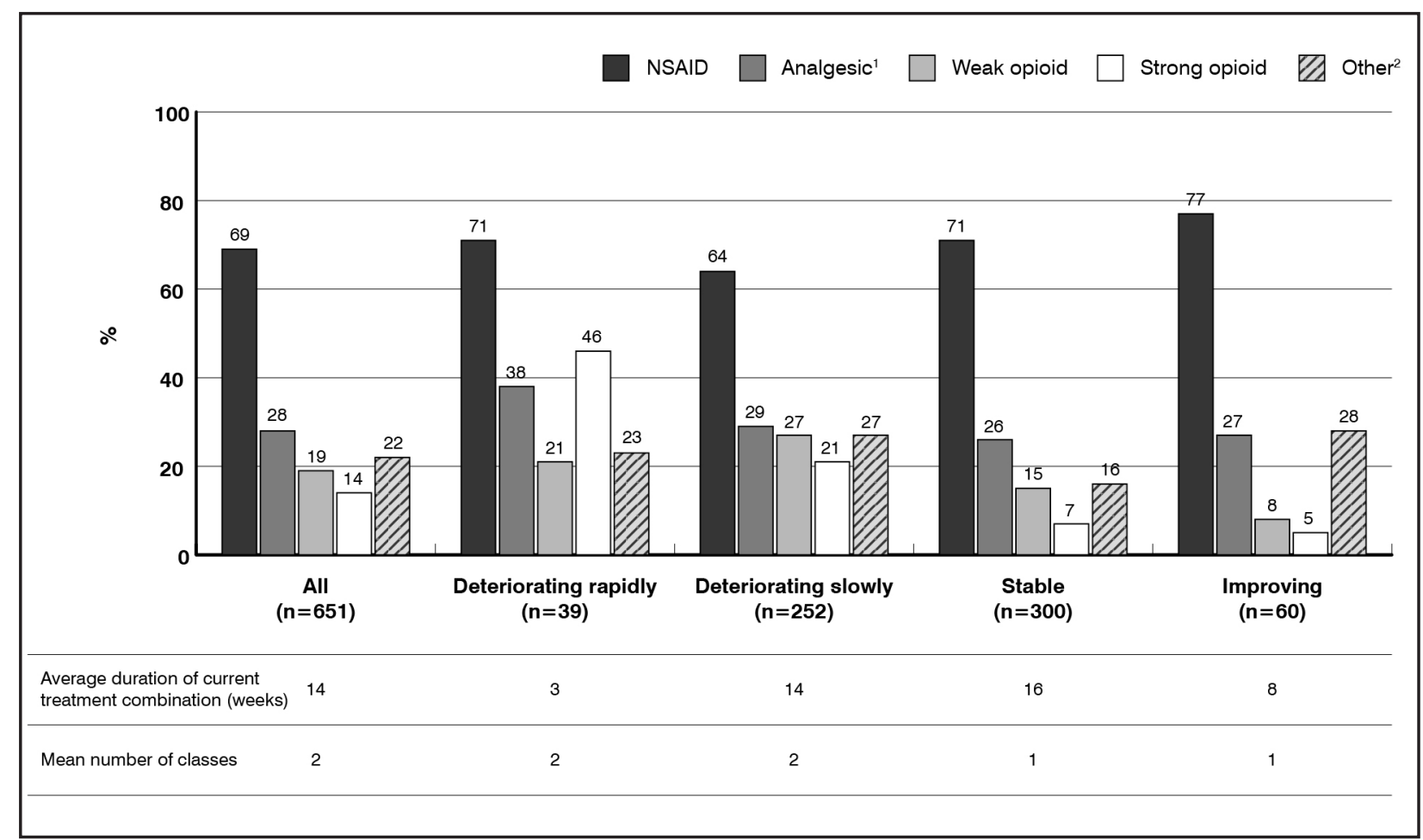

Figure 1. Drug classes by disease progression

${ }^{1}$ Non-opioid /non-NSAID

${ }^{2}$ Other include: Corticosteroids, Opioid Combination, Viscosupplements, Glycosaminoglycans, Other Analgesic, Immunosuppressant, Monoclonal antibodies, Antidepressants, Anticonvulsant, Disease modifying antirheumatic drugs (DMARD), Bisphosphonate

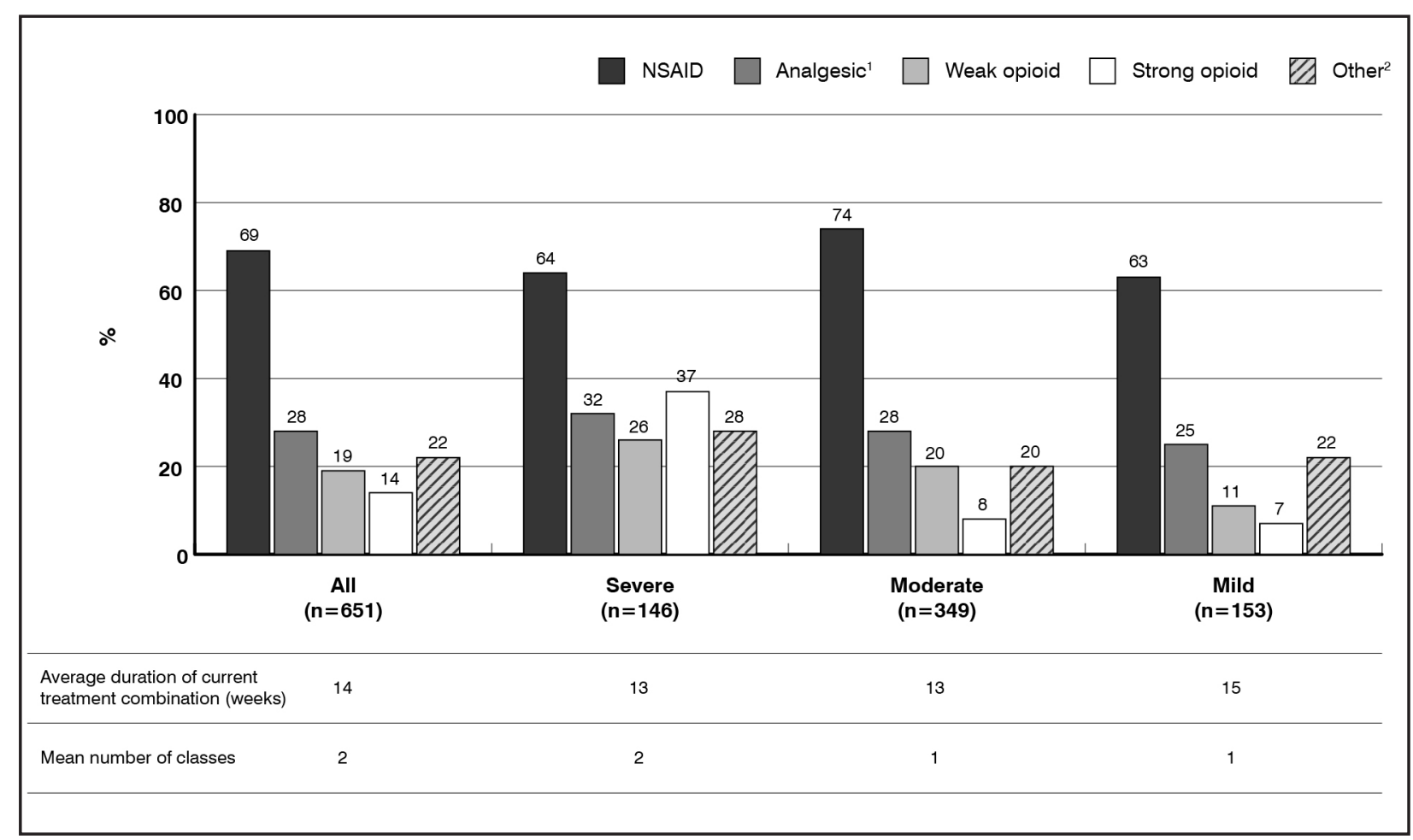

Figure 2. Drug classes by disease severity

${ }_{1}^{1}$ Non-opioid /non-NSAID

2 Other include: Corticosteroids, Opioid Combination, Viscosupplements, Glycosaminoglycans, Other Analgesic, Immunosuppressant, Monoclonal antibodies, Antidepressants, Anticonvulsant, Disease modifying antirheumatic drugs (DMARD), Bisphosphonate

except NSAIDs, which still represent the drug class mostly used in all groups, with consumption being particularly higher in mild and moderate pain patients (Figure 3 ).

On average, across all patient subgroups about $44 \%$ of patients progressed to a second line, $19 \%$ to a third line and only $2 \%$ progressed to a fourth line of treatment, with a ten- 


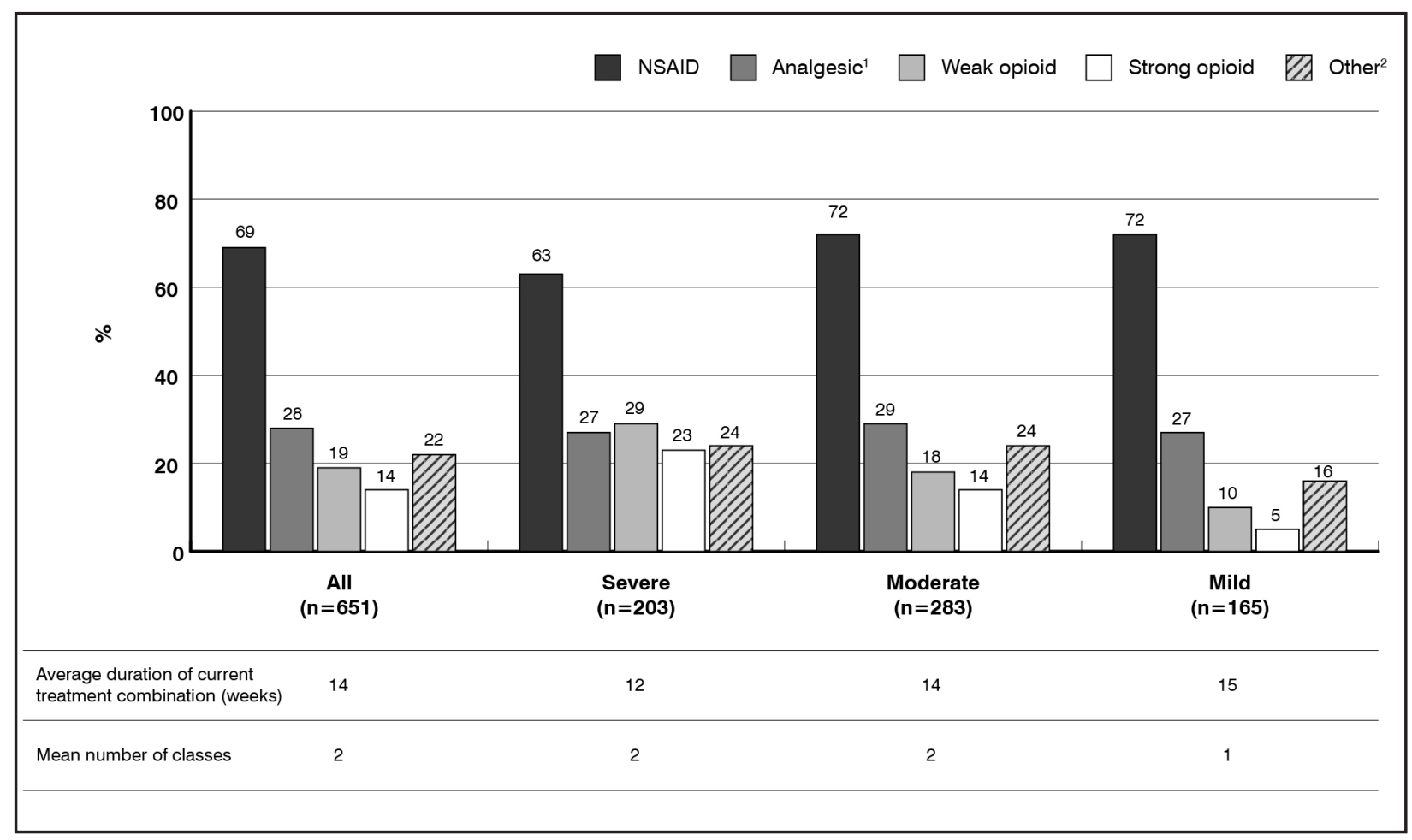

Figure 3. Drug classes by pain severity

${ }^{1}$ Non-opioid /non-NSAID

${ }^{2}$ Other include: Corticosteroids, Opioid Combination, Viscosupplements, Glycosaminoglycans, Other Analgesic, Immunosuppressant, Monoclonal antibodies, Antidepressants, Anticonvulsant, Disease modifying antirheumatic drugs (DMARD), Bisphosphonate

dency to increase the number of classes and particularly to add weak and strong opioids. In patients whose disease was classified as mild only a minority progressed to second $(26 \%)$, third $(10 \%)$ and fourth $(1 \%)$ line of treatment. Most used treatment classes in mild patients were NSAIDs and analgesics with a very limited number of cases in which both weak and strong opioids were used (in second and third line of treatment) (Supplementary Figure 2).

In moderate patients about $43 \%$ of subjects progressed to a second line, $17 \%$ to a third line and less than $1 \%$ progressed to a fourth line of treatment, with a tendency to added weak and strong opioids . $67 \%$ of patients classified as severe progress to second line, $31 \%$ of patients progressed to third and $7 \%$ to fourth line of treatment; percentage transitions across treatment lines in severe patients were the highest (so severe patients show to be treated with more drugs and more treatment lines than any other severity group). Also, in severe patients there was a wider use of strong opioids, especially in patients progressing to the third and fourth line of treatment. Of note, in severe patients the mean pain score was 7, irrespective of treatment line.

Physicians reported their satisfaction with treatment in relation to 651 patients for which a full treatment regimen was recorded (Supplementary Figure 3). In 26.6\% of cases ( $\mathrm{n}=173$ ) they reported being dissatisfied (this includes Neither satisfied nor dissatisfied; Somewhat dissatisfied; Very dissatisfied). Among these 173 cases, reasons for dissatisfaction were investigated, with physicians reporting that in $37 \%$ of cases they find inadequate response and in $27 \%$ lack of improvement in patient quality of life; another $12 \%$ of dissatisfaction reports can be attributed to side effects (notably local reactions to injections; severity of side effects suffered; number of side effects suffered).

Patients reported their satisfaction with OA treatment(s) (Supplementary Figure 4). In $51 \%$ of cases $(n=114)$ they reported being dissatisfied (this includes Neither satisfied nor dissatisfied; Somewhat dissatisfied; Very dissatisfied). Dissatisfaction with treatment seems more marked in the elderly ( $\geq 74$ years old) $(64 \%)$, obese patients $(65 \%)$, and in severe patients as assessed by both patients (81\%) and physicians $(69 \%)$.

Patients reported on OA-related surgeries (Supplementary Figure 5). The average incidence of surgery was $14 \%$ in all patients $(n=242)$, with higher percentages reported by patients in the oldest age group ( $24 \%$ ), by obese (BMI $>30$ ) vs non obese patients ( $32 \%$ vs $10 \%$ ), by patients reporting severe pain $(25 \%)$, and by patients classified as severe according to both patient's (31\%) and physician's (26\%) disease severity assessment. 


\section{Physical Functioning}

Physical functioning was reported by patients using pain, physical function and stiffness scores from the WOMAC, each on a scale 0-10 (Figure 4). In the total population, patients classified as severe, according to physician's and patient's disease severity assessment, reported WOMAC score 19.8 and 18.9, respectively. In addition, a higher WOMAC score was reported in elderly (age 74-89) and obese (BMI>30) patients, 16.1 and 15.9, respectively.

Mobility limitation, need for a walking aid, need for help with daily activities, and suffering a fall were assessed by patient responses to stand-alone questions related to functional limitations.

Among 239 patients, $141(59 \%)$ reported that OA had an impact on physical mobility which seems to affect mostly patients in older age cohorts (63-73 and 74-89) with impairment reported by $67 \%$ and $83 \%$ of patients, respectively. Higher rate of mobility limitation was noted in obese patients (71\%) and, in patients with moderate and severe disease severity, according to patients' severity assessment (66\% and $88 \%$ respectively) as well as according to physicians' severity assessment (66\% and $83 \%$ respectively) (Supplementary Figure 6).

Patients reported their need help for getting around (Supplementary Figure 7). On average, $29 \%$ reported they need a cane/walking stick, $3 \%$ they need a walking frame, $1 \%$ reported they need a wheelchair. Higher percentages were recorded by patients in the older age class; male patients ( $42 \%$ of them need cane/walking stick), obese patients (in total $59 \%$ of them need some sort of support) and severe patients according to patients' disease severity assessment (48\% need support) as well as according to physicians' severity assessment (52\% need support).

$22 \%$ of 237 patients (about one in five individuals) reported that OA generates the need for some help with daily activities or tasks (Supplementary Figure 8). This seems to affect mostly the elderly (74-89) with external need reported by $48 \%$ of patients, obese patients (BMI $>30$ ) with $36 \%$, patients with longer disease duration with reported $24 \%$, and severe patients according to patients' disease severity assessment (47\%) as well as according to physicians' severity assessment $(46 \%)$.

Patients reported on falls attributed to OA. As shown the average incidence of falls was $21 \%$ in all patients $(n=244)$ with higher percentages reported by patients in the oldest age group ( $21 \%$ ), by women vs men ( $23 \%$ vs $17 \%$ ), by obese (BMI>30) vs non obese patients ( $28 \%$ vs $19 \%$ ), by patients reporting severe pain ( $49 \%$ ), and by patients classified as severe according to both patient's (40\%) and physician's (32\%) disease severity assessment (Supplementary Figure 9).

A total of 62 patients reported on the number of hours per week that family, friends or professional caregivers provided to help care (Supplementary Figure 10). Patients were as-

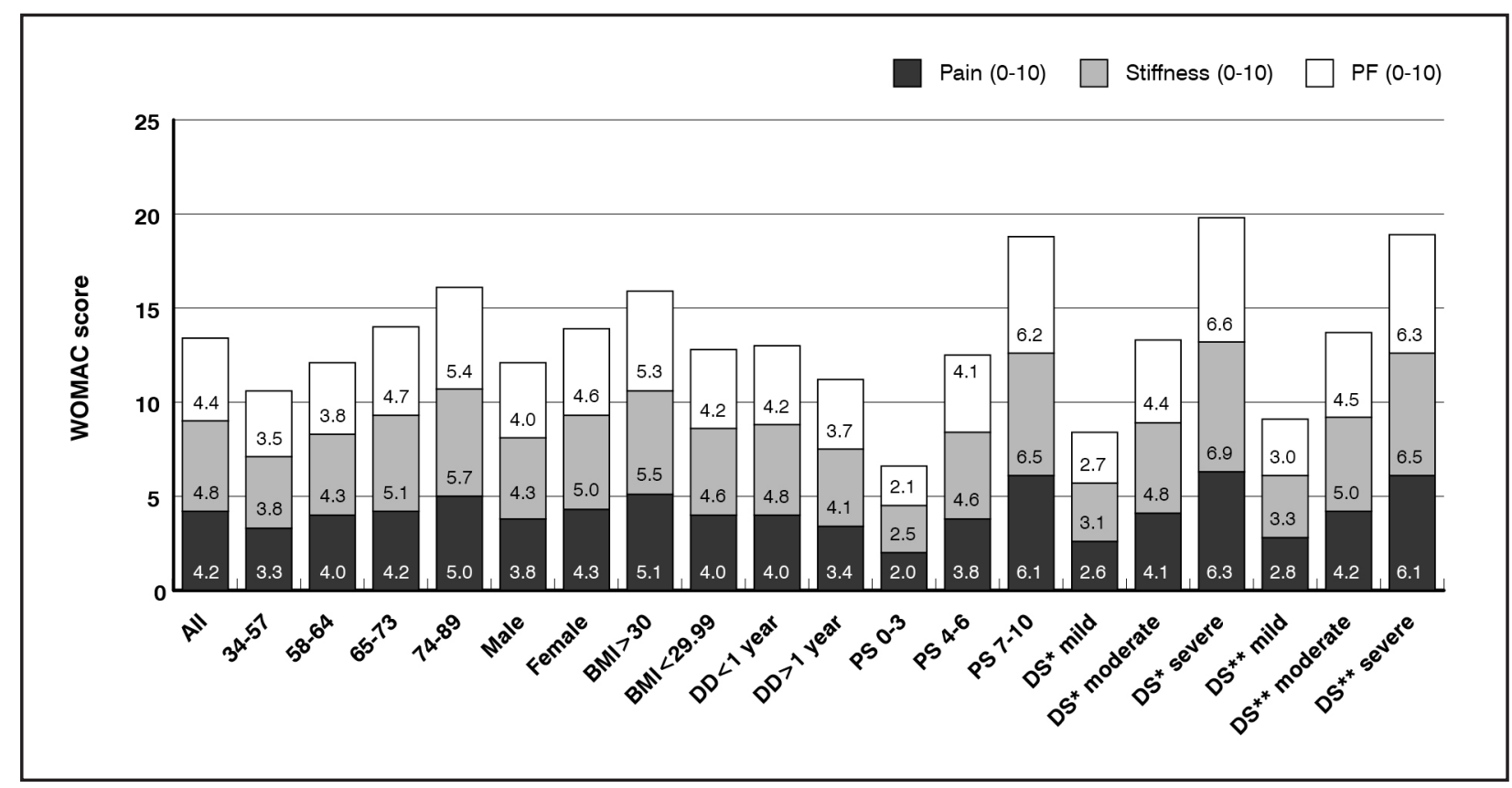

Figure 4. WOMAC scores

$\mathrm{BMI}=$ Body mass of index; DD = Disease duration; DS* = Disease Severity assessed by patient; DS** = Disease severity assessed by physician; PF = Physical functioning; PS = Pain Severity 


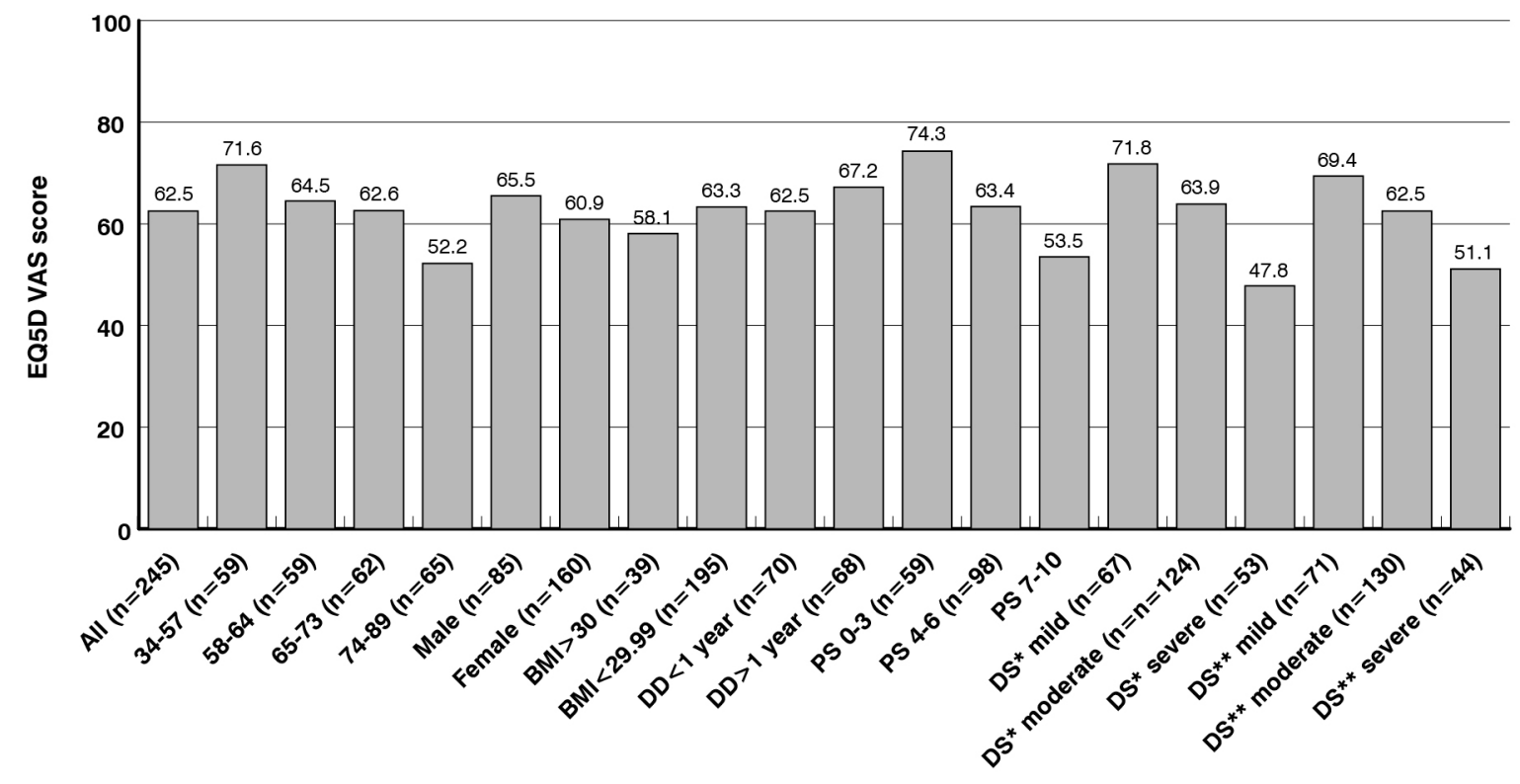

Figure 5. EQ5D VAS score

$\mathrm{BMI}=$ Body mass of index; $\mathrm{DD}=$ Disease duration; $\mathrm{DS} *=$ Disease Severity assessed by patient; $\mathrm{DS}{ }^{\star *}=$ Disease severity assessed by physician $\mathrm{PF}=$ Physical functioning; $\mathrm{PS}=$ Pain Severity

sisted for about 13.6 hours/week on average. Patient groups that required more assistance were those in the $65-73$ age category ( $17.9 \mathrm{hrs} / \mathrm{wk})$, obese patients $(30.3 \mathrm{hrs} / \mathrm{wk})$, patients with higher pain score (20.6 hrs/wk) and patients classified as severe according to both patient's (21.7 hrs/wk) and physician's (17.0 hrs/wk) disease severity assessment.

\section{HRQoL}

HRQoL, assessed by the EQ-5D-5L VAS recorded the patient's self-rated health on a vertical visual analogue scale, where the endpoints are labelled 'The best health you can imagine' and 'The worst health you can imagine' (Figure 5). Patients scored their health at an average of 62.5 with lower scores being recorded for the elderly (52.2), patients with the highest pain severity (53.5) and for patients being classified as severe, according to both physicians (47.8) and patients (51.1) disease severity assessment.

\section{Work Productivity and Daily Activity}

WPAI score on the overall work productivity was collected on 237 subjects (Figure 6). On average OA patients reported their work productivity was impaired by $45 \%$ due to OA problems. Higher WPAI scores were reported by the oldest patients' age group (58\%), by obese patients $(57 \%)$, by patients reporting more severe pain $(66 \%)$ and by patients classified as severe according to both patient's (71\%) and physician's $(67 \%)$ disease severity assessment.

Patients reported the effect of OA on their work productivity over the past 7 days, on a scale $0-10(0=$ no effect; $10=$ completely preventing work) (Supplementary Figure 11$)$. Mean score across all patients was 2.5; higher effect was reported by patients aged 65-73 years (5.0), female (2.9), obese patients (3.3) patients with duration of disease $>1$ year (3.2), patients with highest pain severity score (4.5) and patients classified as severe by both patient's and physician's assessment (score 5.4 and 4.5 , respectively).

73 patients reported having missed on average 3.5 hours of work over the past 7 days, with the highest work loss recorded by patients aged 65-73 years (14 hours), male (6.4 hours), patients with highest pain severity score (12.9) and patients classified as severe by both patient's and physician's assessment (16.8 and 27 hours, respectively) (Supplementary Figure 12).

Almost all patients $(n=237)$ reported on the effect of OA on their ability to perform daily activities over the past 7 days, on a scale $0-10(0=$ no effect; $10=$ completely preventing activity) (Supplementary Figure 13). Mean score across all patients was 4.5; higher effect was reported by patients aged 74-89 years (5.8), obese patients (5.7), patients with highest pain severity score (6.6) and patients classified as severe by both patient's and physician's assessment (score 7.1 and 6.7, respectively). 


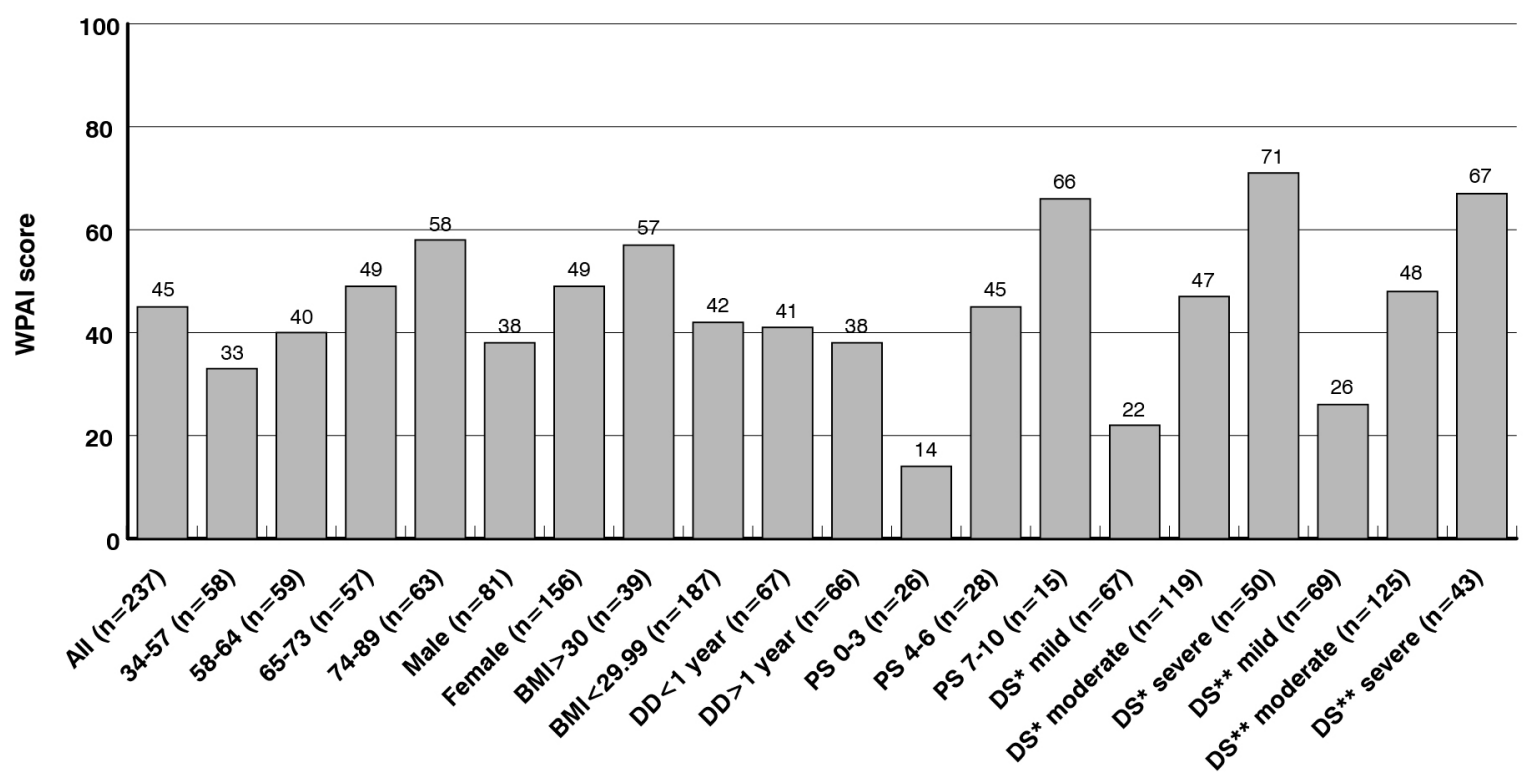

Figure 6. WPAl score

$\mathrm{BMI}=$ Body mass of index; $\mathrm{DD}=$ Disease duration; $\mathrm{DS}^{*}=$ Disease Severity assessed by patient; $\mathrm{DS}^{\star \star}=$ Disease severity assessed by physician; $\mathrm{PF}=$ Physical functioning; $\mathrm{PS}=$ Pain Severity

\section{DISCUSSION}

In this cross-sectional study, Italian patients with severe OA disease severity reported significant burdens that affected multiple aspects of their lives, encompassing reductions in physical functioning, greater treatment needs, reduced HRQoL, and impairments in work productivity and daily activities.

The high prevalence of OA among the elderly is well known [23-25]. Age-related changes occurring in tissues besides articular cartilage may contribute to the development of OA and are much more intense in the presence of obesity [26]. In the current study, burdens affecting OA patients were higher among the elderly and obese individuals.

OA is associated with a significant functional impairment that can result in disability [27]. The results of this study demonstrated that at each level of OA severity, the corresponding magnitude of functional impairment was different, with the greatest impact reported by physicians and patients who rated OA as severe. Patients with severe OA disease were more likely than mild and moderate-patients to report impairment in physical function, pain, stiffness, specific needs such as for a walking aid or help with daily activities, help care and that they suffered a fall. These results are in line with several cross-sectional studies that found a relationship between disease severity and physical function [28-31].

In OA patients, HRQoL is negatively affected $[32,33]$. In this study, HRQoL, as measured by the EQ-5D VAS was lower among patients classified as severe, according to both physician's and patient's disease severity assessment. HRQoL was also lower among patients with higher pain severity. A systemic review by Vitaloni et al. revealed a substantial impact on quality of life (QoL) in patients affected by knee osteoarthritis (KOA). All reviewed studies reported worse QoL in KOA patients when compared to a control group. When females were compared to males, females reported worse QoL. Obesity, as well as lower level of physical activity, were also reported with lower QoL scores [34].

Previous studies have demonstrated an association between OA and reduced work productivity and the ability to perform daily activities $[35,36]$ and such impairments were also reported by patients in this study. These impairments, as measured by the WPAI score, were associated with higher disease severity and pain levels. Work productivity and daily activity impairments were higher in patients with highest pain severity score and in patients classified as severe by both patient's and physician's assessment. This finding is consistent with the results of other studies in OA showing that pain and disease severity has a negative association with employment status and productivity, including both absenteeism and presenteeism [30,31].

The current study revealed a higher medication burden among patients with OA, and a greater proportion of these patients who were prescribed potent drugs. Even if NSAIDs are 
considered first-line treatment for OA, opioids were the most frequently prescribed medication class in this Italian patients' population. There was a higher prescribing rate of strong and weak opioids in patients with severe pain as well as in patients whose OA was classified as severe by both patient's and physician's assessment. This high rate of opioid prescribing is in accord with other studies that have shown opioids to be the most frequently prescribed pain-related medications in patients with OA [37]. In particular patients with severe disease reported use of two treatment regimens for OA. Additionally, physicians reported treatment dissatisfaction related to inadequate response and lack of improvement in patient quality of life while dissatisfaction from patients seems more marked in the elderly, obese patients, and severe patients.

Across all classes of prescription medications, approximately only one third of OA patients reported being very or extremely satisfied with their current medication, presenting some relevant problems, such as poor compliance / adherence to the treatments. Kingsbury et al. showed that $42 \%$ of Italian OA patients considered themselves to be fully adherent to their medication [36] and, the most common classes of prescription medication were NSAIDs and COX-2 inhibitors [36,38].

Healthcare resource utilization relative to individuals with OA is responsible for a substantial proportion of the economic burden of OA. These resources include not only pharmacologic and other therapies related for OA, but also management of treatment-related complications and surgery/ rehabilitation [18,39-42]. In the current study, OA patients classified as severe reported higher percentages of OA-related surgeries. In Italy during 2015 were performed around 181.738 surgeries $(56.3 \%$ relates to the hip, $38.6 \%$ knee, $3.9 \%, 0.3 \%$ and $0.9 \%$ respectively shoulder, ankle and other joints). Since 2001 to 2015, the average annual increase in the number of total interventions was $4.1 \%$ [43]. The incremental healthcare costs of people with osteoarthritis compared to those without the disease reaches a maximum when osteoarthritisrelated surgery becomes necessary [15]. The main direct health-related cost driver is the total joint arthroplasty [44]. The socioeconomic burden of total joint arthroplasties generated additional costs due to relapses, need for intervention review, adverse events (e.g. infections, thrombosis, and pulmonary embolisms) and subsequently to the rehabilitation process, in hospital and at home [45]. The steady increase, year after year, of surgery incidence led costs to grow [45-47] and this is expected also for the next future, in an ageing population [46].

As in previous studies that reported high rates of healthcare utilization among OA patients in terms of number of visits to a health care provider and hospitalizations $[18,29,38,48]$, in the current study patients with OA utilized outpatient resources including medical and other health care professional (HCP) visits. This resource utilization was higher in patients with higher pain severity and in patients classified as severe by both patient's and physician's assessment. In Italy hospitalization represented $40 \%$ of the medical costs and among different admissions, those in rehabilitative care were the most expensive due to a stay in hospital prolonged up to 50 days in patients operated for arthroprothesis [18].

Interpretation and extrapolation of these results are subject to study limitations. These limitations include study participation based on agreement by physicians and patients. It is therefore possible that individuals who participated may have characteristics and perceptions different from those who refused to participate, thereby introducing selection bias and reducing generalizability. In addition, we are unable to attribute linked causality between OA and reported outcomes, since it is possible that co-morbid conditions and other factors may have contributed in part to the differences described. Furthermore, statistical comparisons were not conducted between the patient groups limiting the analysis of relevant differences.

\section{CONCLUSION}

This real-world-cross-sectional study demonstrated an increase in the scoring for all dimensions of QoL as well as in the use of resources as OA disease severity worsened. The burdens were higher among severe patients versus moderate and mild patients. Novel therapeutic agents that improve OA symptom management may lead to significant healthcare resource utilization savings.

\section{Funding}

This study was sponsored by Pfizer and Eli Lilly and Company. The analysis was conducted independently by Adelphi Real World. All authors actively collaborated on the study design and interpretation of results; contributed in writing this paper; and have provided final approval of the submitted version.

\section{Conflicts of interest}

PB and MDS are consultants of Regulatory Pharma Net srl. $\mathrm{JJ}, \mathrm{MB}$, and $\mathrm{JJ}$ are employees of Adelphi Real World. 


\section{REFERENCES}

1. Roman-Blas JA, Castaneda S, Largo R, et al. Osteoarthritis associated with estrogen deficiency. Arthritis Res Ther 2009; 11:241; https://doi.org/10.1186/ar2791

2. Herrero-Beaumont G, Roman-Blas JA, Castaneda S, et al. Primary osteoarthritis no longer primary: three subsets with distinct etiological, clinical, and therapeutic characteristics. Semin Arthritis Rheum 2009;39:71-80; https://doi. org/10.1016/j.semarthrit.2009.03.006

3. Arden N, Nevitt MC. Osteoarthritis: epidemiology. Best Pract Res Clin Rheumatol 2006;20:3-25; https://doi. org/10.1016/j.berh.2005.09.007

4. Loeser RF. The Role of Aging in the Development of Osteoarthritis. Trans Am Clin Climatol Assoc 2017;128:44-54

5. Neogi T. The epidemiology and impact of pain in osteoarthritis. Osteoarthr Cartil 2013;21:1145-53; https://doi. org/10.1016/j.joca.2013.03.018

6. O’Neill TW, McCabe PS, McBeth J. Update on the epidemiology, risk factors and disease outcomes of osteoarthritis. Best Pract Res Clin Rheumatol 2018;32:312-26; https://doi.org/10.1016/j.berh.2018.10.007

7. Johnson SR, Archibald A, Davis AM, et al. Is self-reported improvement in osteoarthritis pain and disability reflected in objective measures? J Rheumatol 2007;34:159-164

8. Hochberg MC, Altman RD, April KT, et al. American College of Rheumatology 2012 Recommendations for the use of nonpharmacologic and pharmacologic therapies in osteoarthritis of the hand, hip, and knee. Arthritis Care Res 2012;64:465-474; https://doi.org/10.1002/acr.21596

9. Punchak S, Goodyer LI, Miskelly F. Use of an electronic monitoring aid to investigate the medication pattern of analgesics and non-steroidal anti-inflammatory drugs prescribed for osteoarthritis. Rheumatology (Oxford) 2000;39:448-449; https://doi.org/10.1093/rheumatology/39.4.448

10. Sadosky AB, Bushmakin AG, Cappelleri JC, et al. Relationship between patient-reported disease severity in osteoarthritis and self-reported pain, function and work productivity. Arthritis Research \& Therapy 2010;12:R162; https://doi.org/10.1186/ar3121

11. Ricci JA, Stewart WF, Chee E, et al. Pain exacerbation as a major source of lost productive time in US workers with arthritis. Arthritis Rheum 2005;53:673-81; https://doi.org/10.1002/art.21453

12. Hutchings A, Calloway M, Choy E, et al. The Longitudinal Examination of Arthritis Pain (LEAP) study: relationships between weekly fluctuations in patient-rated joint pain and other health outcomes. J Rheumatol 2007;34:2291-300

13. Wang SX, Ganguli AX, Bodhani A, et al. Healthcare resource utilization and costs by age and joint location among osteoarthritis patients in a privately insured population. J Med Econ 2017;20:1299-306; https://doi.org/10.1080/1 3696998.2017.1377717

14. Safiri S, Kolahi A, Smith E, et al. Global, regional and national burden of osteoarthritis 1990-2017: a systematic analysis of the Global Burden of Disease Study 2017. Annals of the Rheumatic Diseases 2020;79:819-828; https:// doi.org/10.1136/annrheumdis-2019-216515

15. Puig-Junoy J, Ruiz ZA. Socio-economic costs of osteoarthritis: a systematic review of cost-of-illness studies. Semin Arthritis Rheum 2015;44:531-41; https://doi.org/10.1016/j.semarthrit.2014.10.012

16. Russo S, Landi S, Landa P. Management of knee osteoarthritis in Italy. A cost-utility analysis of Platelet-Rich-Plasma dedicated kit versus Hyaluronic acid for the intra-articular treatment of knee OA. SRRN Electronic journal 2017

17. Direzione Generale della Programmazione sanitaria Ufficio VI. Italian Hospital Discharges 2014. Rapporto annuale sull'attività di ricovero ospedaliero Dati SDO 2014, Ministero della salute, 2015

18. Leardini G, Salaffi F, Caporali R, et al. Italian Group for Study of the Costs of Arthritis, Direct and indirect costs of osteoarthritis of the knee. Clin Exp Rheumatol 2004;22:699-706

19. Berto P, Aiello A. Impatto epidemiologico, economico e sociale dell'osteoartrosi in Italia. IHPB Anno X; speciale 2020

20. Anderson P, Benford M, Harris N, et al. Real-world physician and patient behaviour across countries: disease-specific programmes a means to understand. Curr Med Res Opin 2008;24:3063-72; https://doi. org/10.1185/03007990802457040

21. Bellamy N. WOMAC 3.1 Osteoarthritis Index User Guide XI. July 2016. Available at https://www.womac.com/ womac/index.htm (last accessed December 2019)

22. Rabin R, de Charro F. EQ-5D: a measure of health status from the EuroQol group. Ann Med 2001;33:337-43; https:// doi.org/10.3109/07853890109002087 
23. Garstang SV, Stitik TP. Osteoarthritis: epidemiology, risk factors, and pathophysiology. Am J Phys Med Rehabil 2006; 85: S2-11; https://doi.org/10.1097/01.phm.0000245568.69434.1a

24. Quintana JM, Arostegui I, Escobar A, et al. Prevalence of knee and hip osteoarthritis and the appropriateness of joint replacement in an older population. Arch Intern Med 2008;168:1576-84; https://doi.org/10.1001/archinte.168.14.1576

25. Dagenais S, Garbedian S, Wai EK. Systematic review of the prevalence of radiographic primary hip osteoarthritis. Clin Orthop Relat Res 2009;467:623-37; https://doi.org/10.1007/s11999-008-0625-5

26. Li Y, Wei X, Zhou J, et al. The age-related changes in cartilage and osteoarthritis. Biomed Res Int 2013;2013:916530; https://doi.org/10.1155/2013/916530

27. Kloppenburg M, Berenbaum F. Osteoarthritis year in review 2019: epidemiology and therapy. Osteoarthritis and Cartilage 2020;28:242-8; https://doi.org/10.1016/j.joca.2020.01.002

28. Creamer P, Lethbridge-Cejku M, Hochberg MC. Factors associated with functional impairment in symptomatic knee osteoarthritis. Rheumatology 2000;39:490-496; https://doi.org/10.1093/rheumatology/39.5.490

29. Gore M, Tai KS, Sadosky A, et al. Clinical comorbidities, treatment patterns, and direct medical costs of patients with osteoarthritis in usual care: a retrospective claims database analysis. Journal of Medical Economics 2011;14:497507; https://doi.org/10.3111/13696998.2011.594347

30. Bushmakin AG, Cappelleri JC, Taylor-Stokes G, et al. Relationship between patient-reported disease severity and other clinical outcomes in osteoarthritis: a European perspective. J Med Econ 2011;14:381-9; https://doi.org/10.3 $111 / 13696998.2011 .583703$

31. Jackson J, Iyer R, Mellor J, et al. The Burden of Pain Associated with Osteoarthritis in the Hip or Knee from the Patient's Perspective: A Multinational Cross-Sectional Study. Adv Ther 2020;37:3985-99; https://doi.org/10.1007/ s12325-020-01445-4

32. Dominick KL, Ahern FM, Gold CH, et al. Health-related quality of life and health service use among older adults with osteoarthritis. Arthritis Rheum 2004;51:326-31; https://doi.org/10.1186/1477-7525-2-5

33. Kawano MM, Araujo IL, Castro MC, et al. Assessment of quality of life in patients with knee osteoarthritis. Acta Ortop Bras 2015;23:307-10; https://doi.org/10.1590/1413-785220152306150596

34. Vitaloni M, Botto-van BA, Sciortino Contreras RM, et al. Global management of patients with knee osteoarthritis begins with quality of life assessment: a systematic review. BMC Musculoskelet Disord 2019;20:493; https://doi. org/10.1186/s12891-019-2895-3

35. Dibonaventura MD, Gupta S, McDonald M, et al. Evaluating the health and economic impact of osteoarthritis pain in the workforce: results from the National Health and Wellness Survey. BMC Musculoskelet Disord 2011;12:83; https://doi.org/10.1186/1471-2474-12-83

36. Kingsbury SR, Gross HJ, Isherwood G, et al. Osteoarthritis in Europe: impact on health status, work productivity and use of pharmacotherapies in five European countries. Rheumatology (Oxford) 2014;53:937-47; https://doi. org/10.1093/rheumatology/ket463

37. White AG, Birnbaum HG, Janagap C, et al. Direct and indirect costs of pain therapy for osteoarthritis in an insured population in the United States. J Occup Environ Med 2008;50:998-1005; https://doi.org/10.1097/ JOM.0b013e3181715111

38. Degli Esposti L, Di Martino M, Didoni G, et al. Pharmacoutilization of anti-inflammatory and gastroprotective drugs in patients with osteoarthritis: comparison between COXIBs and conventional NSAIDs by using administrative records. Farmeconomia Health economics and therapeutic pathways 2006;7:247-254; https://doi.org/10.7175/ fe.v7i4.259

39. Rabenda V, Manette C, Lemmens R, et al. Direct and indirect costs attributable to osteoarthritis in active subjects. J Rheumatol 2006;33:1152-8

40. Gupta S, Hawker GA, Laporte A, et al. The economic burden of disabling hip and knee osteoarthritis (OA) from the perspective of individuals living with this condition. Rheumatology (Oxford) 2005;44:1531-7; https://doi. org/10.1093/rheumatology/kei049

41. Gabriel SE, Crowson CS, Campion ME, et al. Direct medical costs unique to people with arthritis. J Rheumatol 1997;24:719-25

42. Dunn JD, Pill MW. A claims-based view of health care charges and utilization for commercially insured patients with osteoarthritis. Manag Care 2009;18:44-50

43. Torre M. Italian Arthroplasty register 2017. Progetto Registro Italiano ArtroProtesi. Verso l'operatività. Secondo Report, RIAP, ISS Istituto Superiore di Sanità, 2017. Available at: https://www.epicentro.iss.it/muscoloscheletriche/ pdf/PSE_Volume_RIAP_2017.pdf 
44. Piscitelli P, Iolascon G, Di Tanna G, et al. Socioeconomic burden of total joint arthroplasty for symptomatic hip and knee osteoarthritis in the Italian population: a 5-year analysis based on hospitalization records. Arthritis Care Res (Hoboken) 2012;64:1320-7; https://doi.org/10.1002/acr.21706

45. Chen A, Gupte C, Akhtar K, Smith P, Cobb J. The global economic cost of osteoarthritis: how the UK compares. Arthiritis 2012:698-709; https://doi.org/10.1155/2012/698709

46. Hiligsmann M, Cooper C, Arden N, et al. Health economics in the field of osteoarthritis: an expert's consensus paper from the European Society for Clinical and Economic Aspects of Osteoporosis and Osteoarthritis (ESCEO). Semin Arthritis Rheum 2013; 43:303-13; https://doi.org/10.1007/s40520-017-0780-1

47. White AG, Birnbaum HG, Janagap CC, et al. Direct and indirect costs of pain therapy for osteoarthritis in an insured population in the United States. J Occup Environ Med 2008;50:998-1005; https://doi.org/10.1097/ JOM.0b013e3181715111

48. Mapel DW, Shainline M, Paez K, et al. Hospital, pharmacy, and outpatient costs for osteoarthritis and chronic back pain. J Rheumatol 2004;31:573-83 
Farmeconomia. Health economics and therapeutic pathways 2021; 22(1): 1-7

https://doi.org/10.7175/fe.v22i1.1496

\section{Osteoarthritis in Italy: Impact on Health-Related Quality of Life and Health Care Resources}

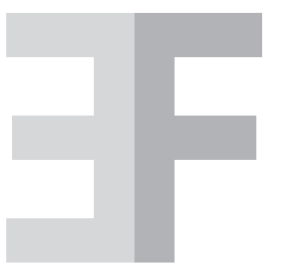

SUPPLEMENTARY FILES

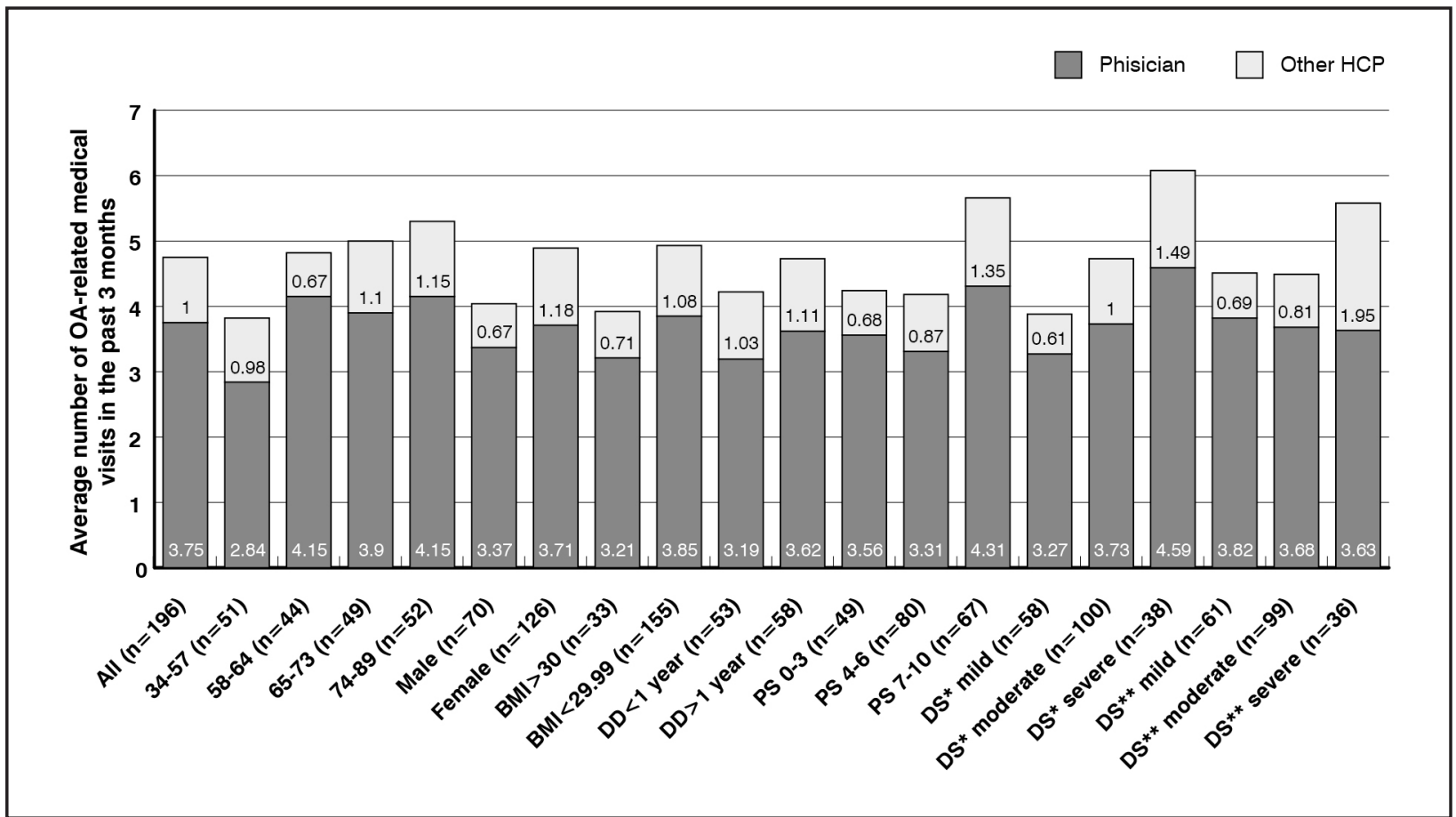

Figure 1. Average number of OA-related medical visits in the past 3 months

$\mathrm{BMI}=$ Body mass of index; DD = Disease duration; $\mathrm{DS}^{\star}=$ Disease Severity assessed by patient; $\mathrm{DS}^{\star \star}=$ Disease Severity assessed by physician; $\mathrm{HCP}=$ Health Care Professional; PS = Pain Severity

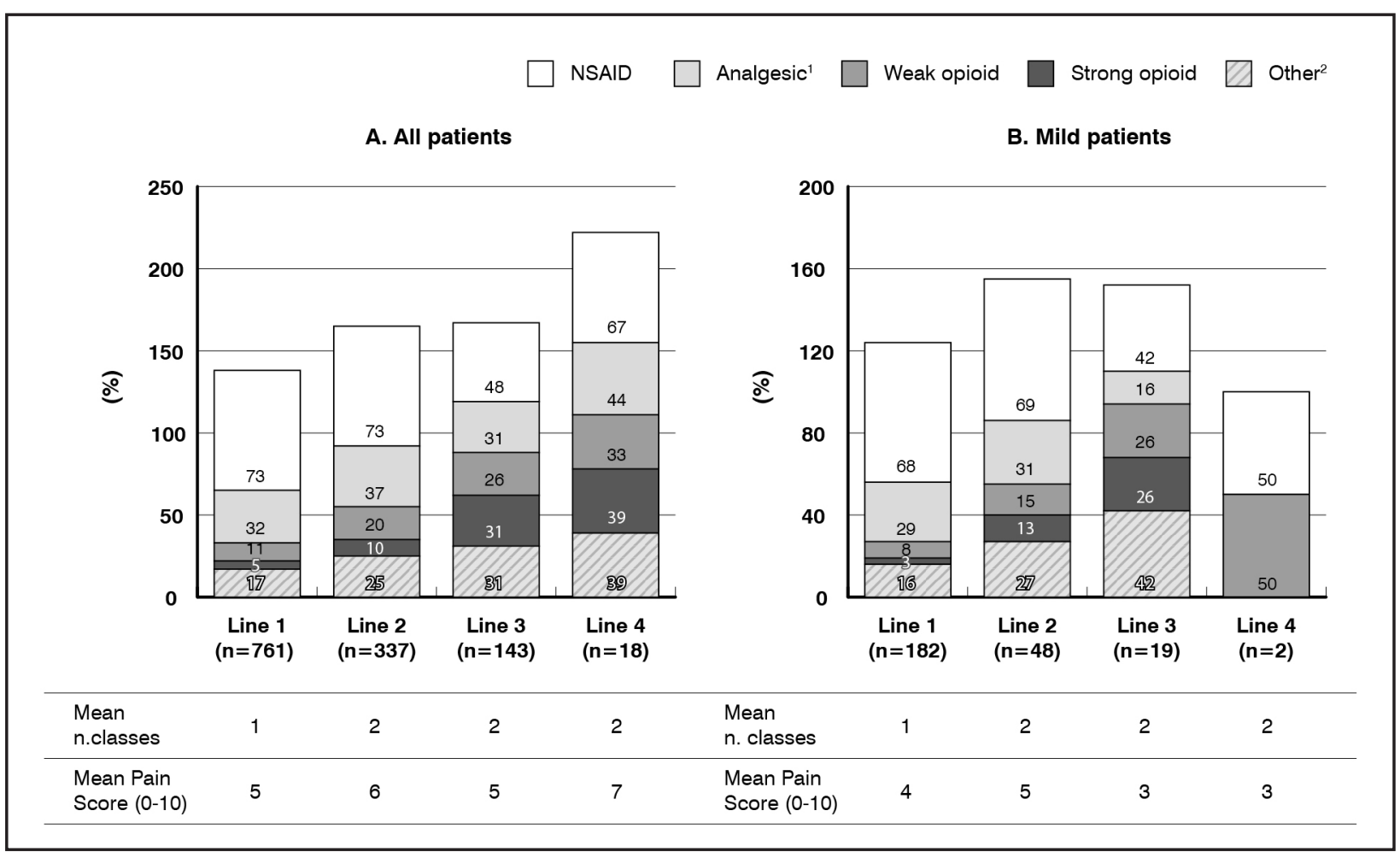




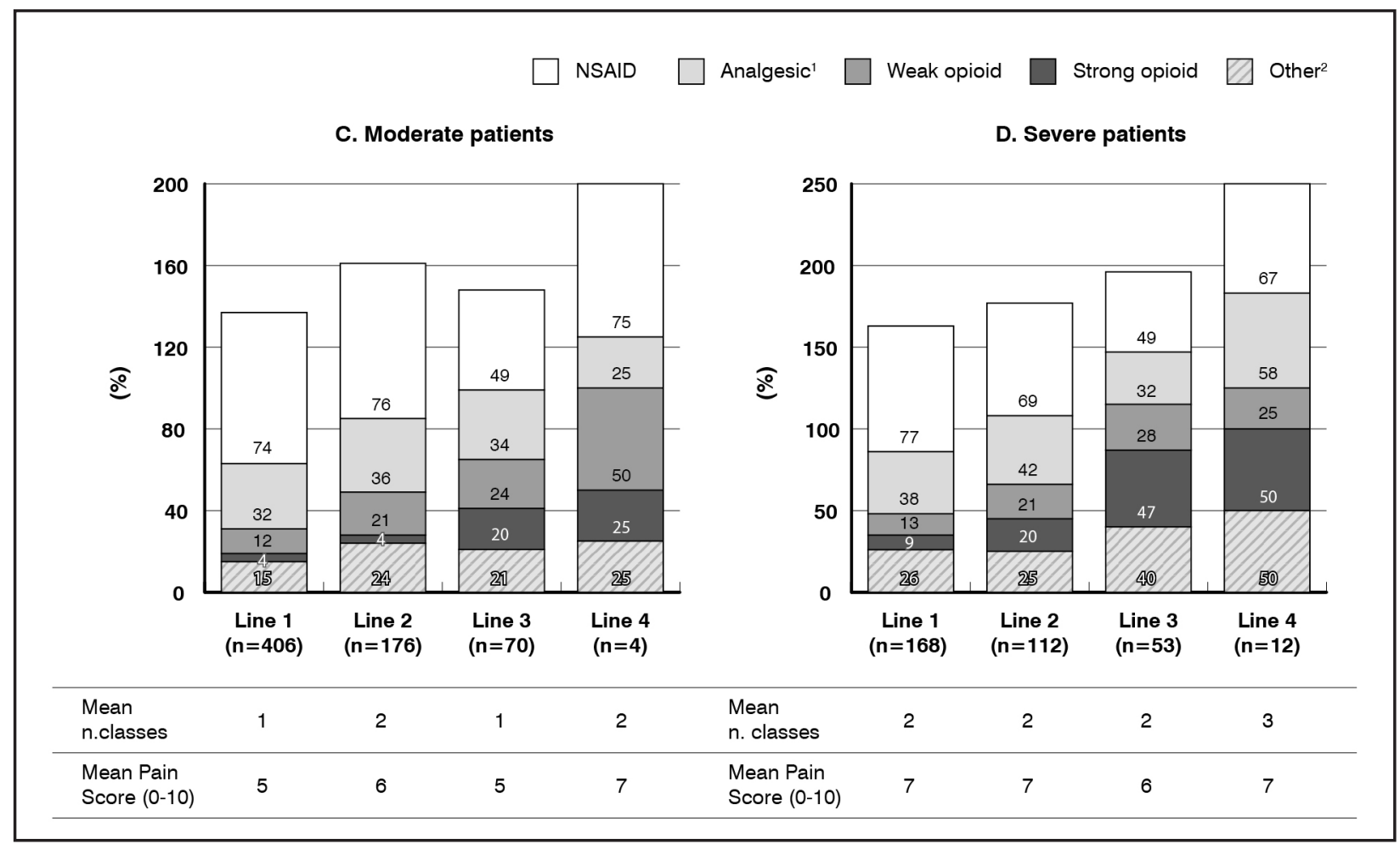

Figure 2. Treatment history by disease severity 1 (non-opioid /non-NSAID)

${ }^{2}$ Other include: Corticosteroids, Opioid Combination, Viscosupplements, Glycosaminoglycans, Other Analgesic, Immunosuppressant, Monoclonal antibodies, Antidepressants, Anticonvulsant, Disease modifying antirheumatic drugs (Dmard), Bisphosphonate

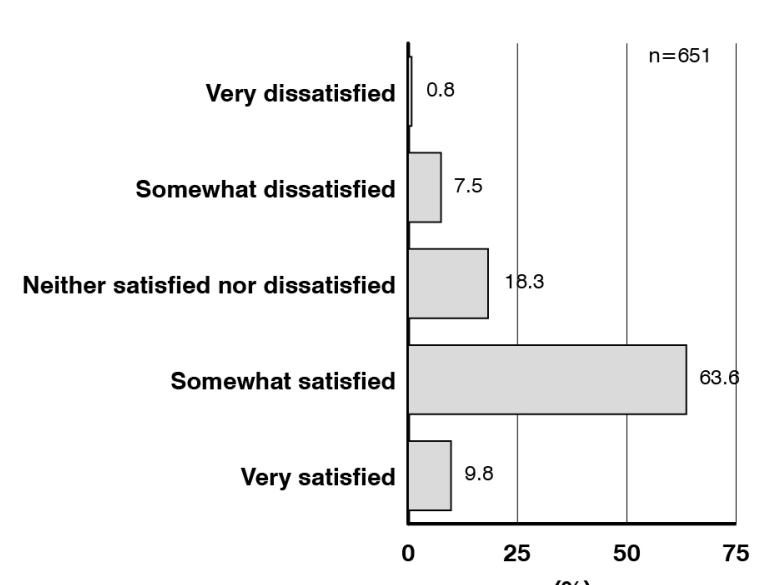

(\%)

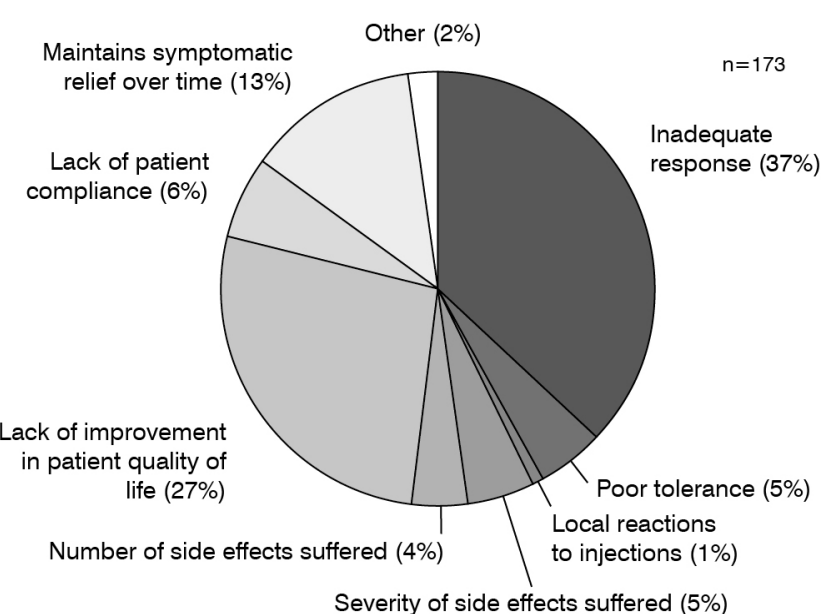

Severity of side effects suffered $(5 \%)$

Figure 3. 3A Physician's satisfaction with treatment ( $n=651)$; 3B Reason for dissatisfaction $(n=173)$ 


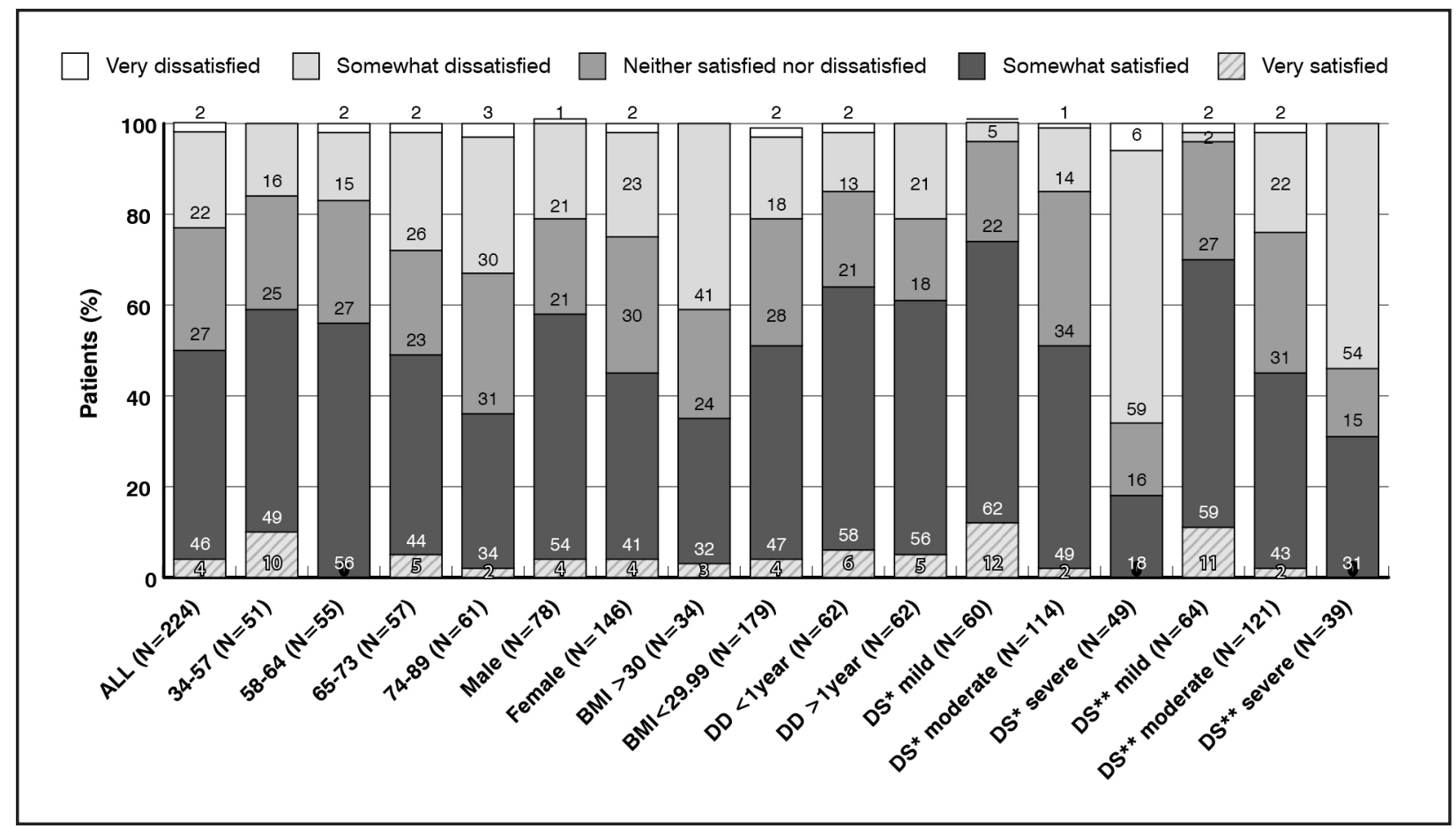

Figure 4. Patient's satisfaction with treatment

$\mathrm{BMI}=$ Body mass of index; DD = Disease duration; $\mathrm{DS}^{*}=$ Disease Severity assessed by patient; $\mathrm{DS}{ }^{\star *}=$ Disease Severity assessed by physician

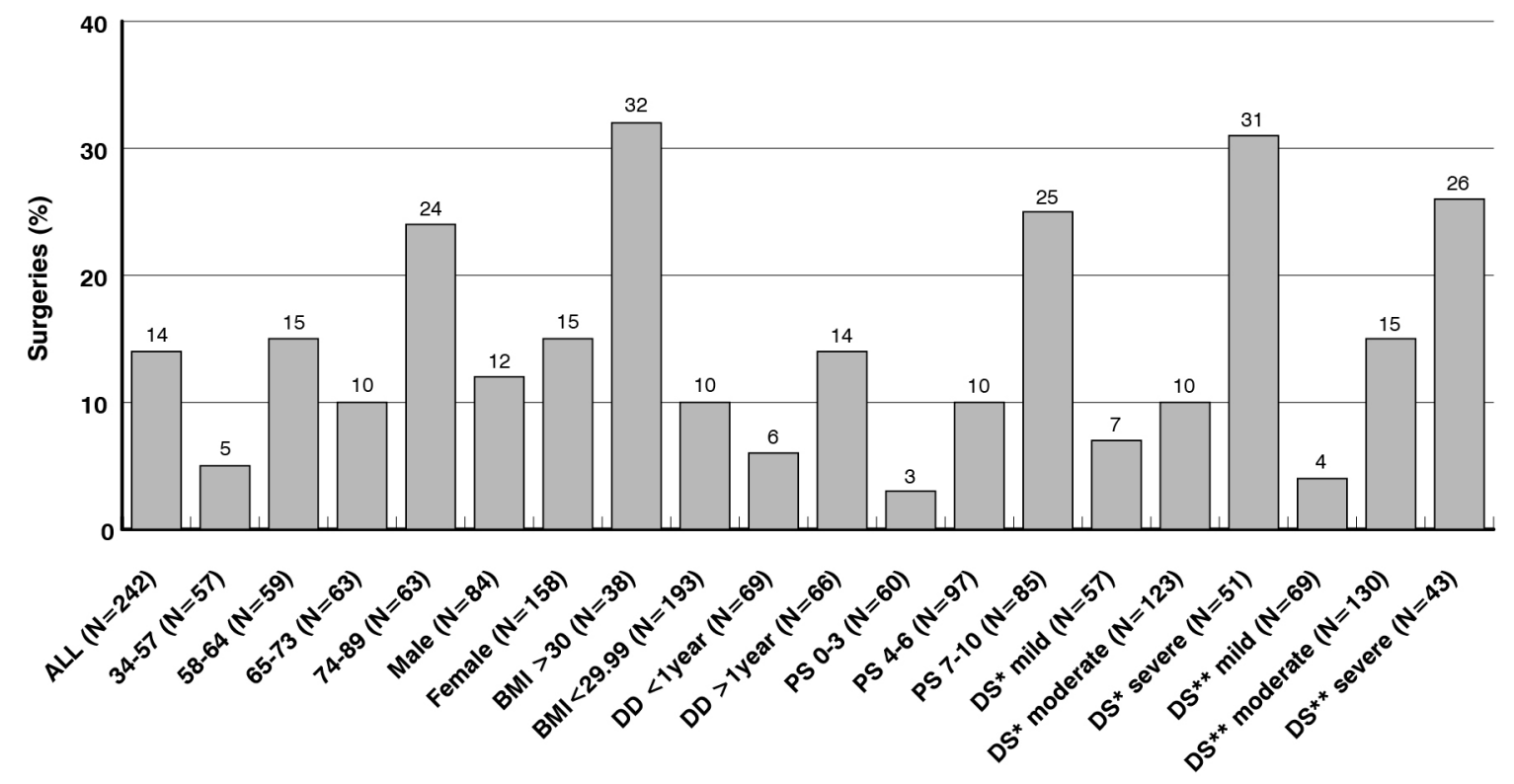

Figure 5. Surgeries due to $O A$

$\mathrm{BMI}=$ Body mass of index; DD = Disease duration; DS* $=$ Disease Severity assessed by patient; DS ${ }^{\star \star}=$ Disease Severity assessed by physician; PS = Pain Severity 


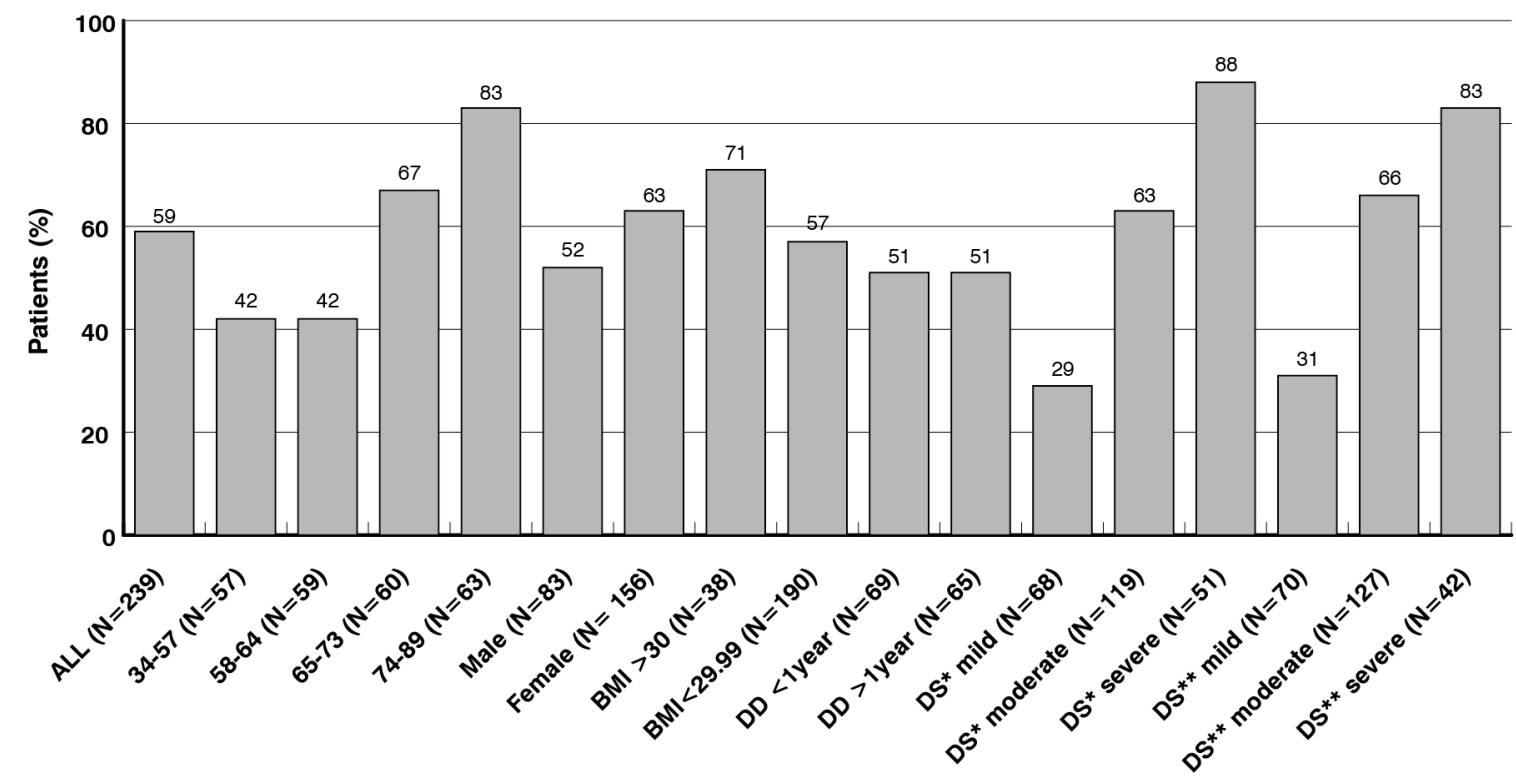

Figure 6. Impact on physical mobility

$\mathrm{BMI}=$ Body mass of index; DD = Disease duration; $\mathrm{DS}^{\star}=$ Disease Severity assessed by patient; DS ${ }^{\star \star}=$ Disease Severity assessed by physician

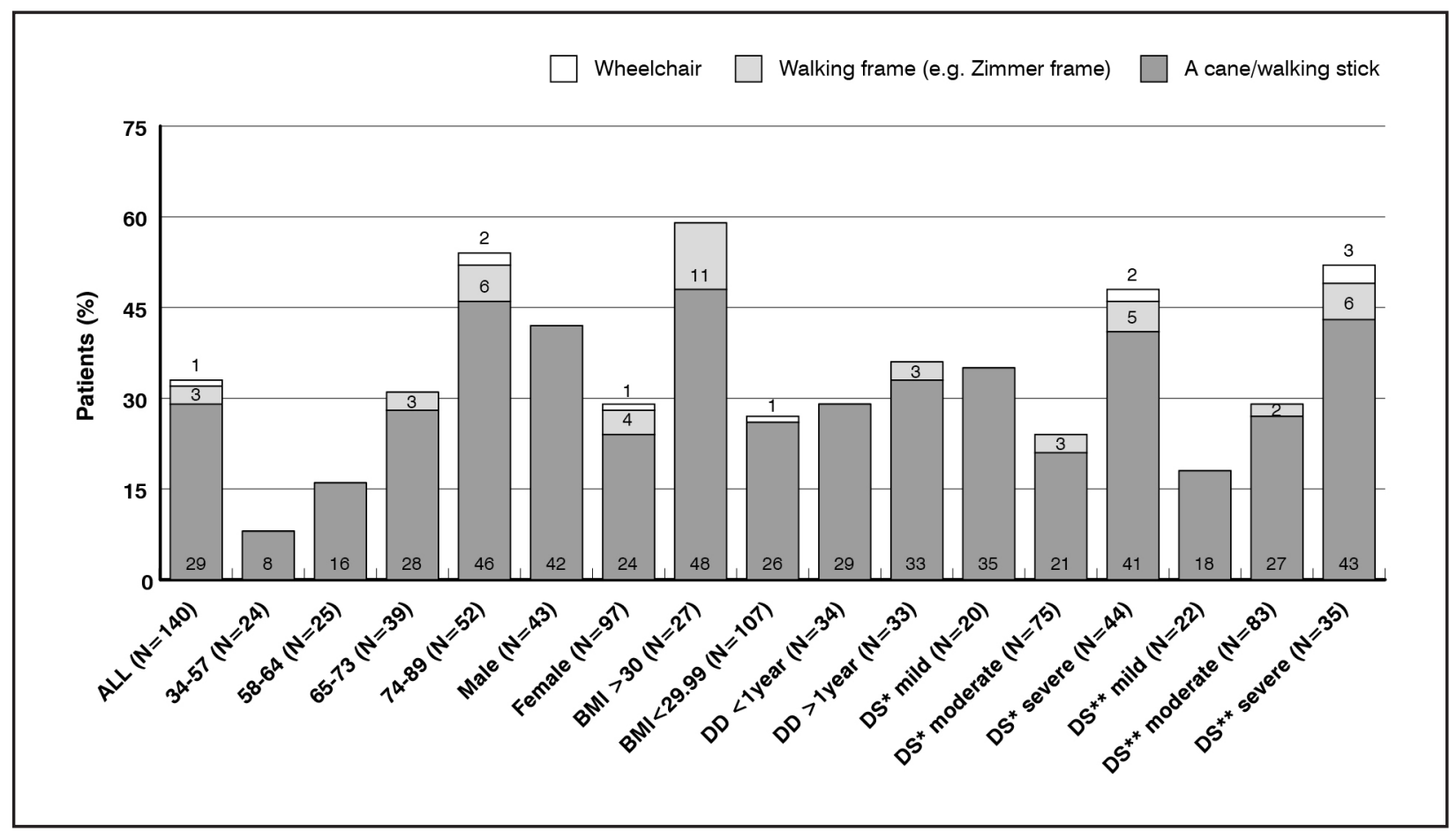

Figure 7. Use of walking aids

$\mathrm{BMI}=$ Body mass of index; $\mathrm{DD}=$ Disease duration; $\mathrm{DS}^{*}=$ Disease Severity assessed by patient; $\mathrm{DS}{ }^{\star *}=$ Disease Severity assessed by physician 


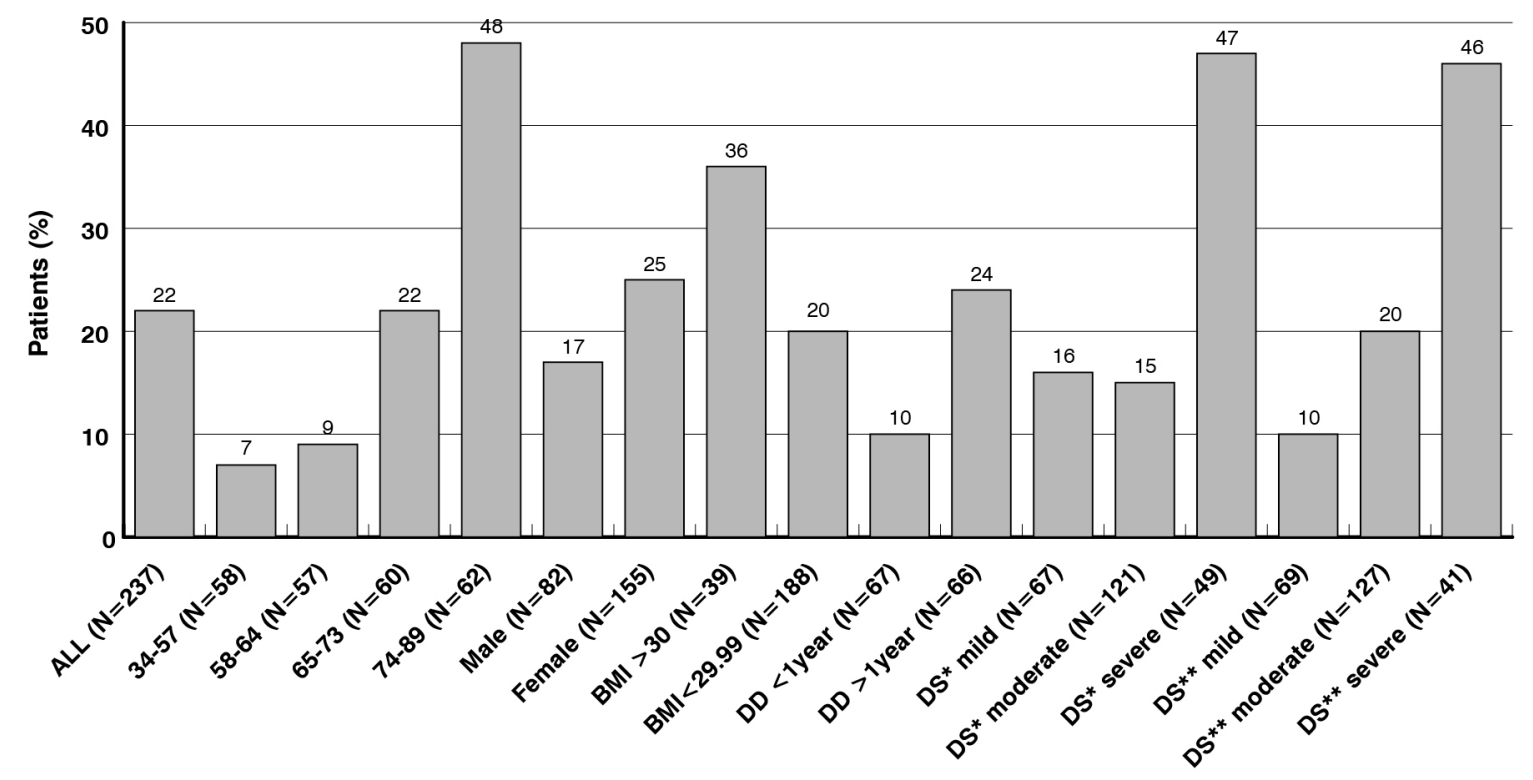

Figure 8. Need for help in daily activity

$\mathrm{BMI}=$ Body mass of index; DD = Disease duration; DS* $=$ Disease Severity assessed by patient; DS** $=$ Disease Severity assessed by physician



Figure 9. Falls

$\mathrm{BMI}=$ Body mass of index; DD = disease duration; $\mathrm{DS}^{*}=$ Disease Severity assessed by patient; DS** $=$ Disease Severity assessed by physician; PS = Pain Severity 


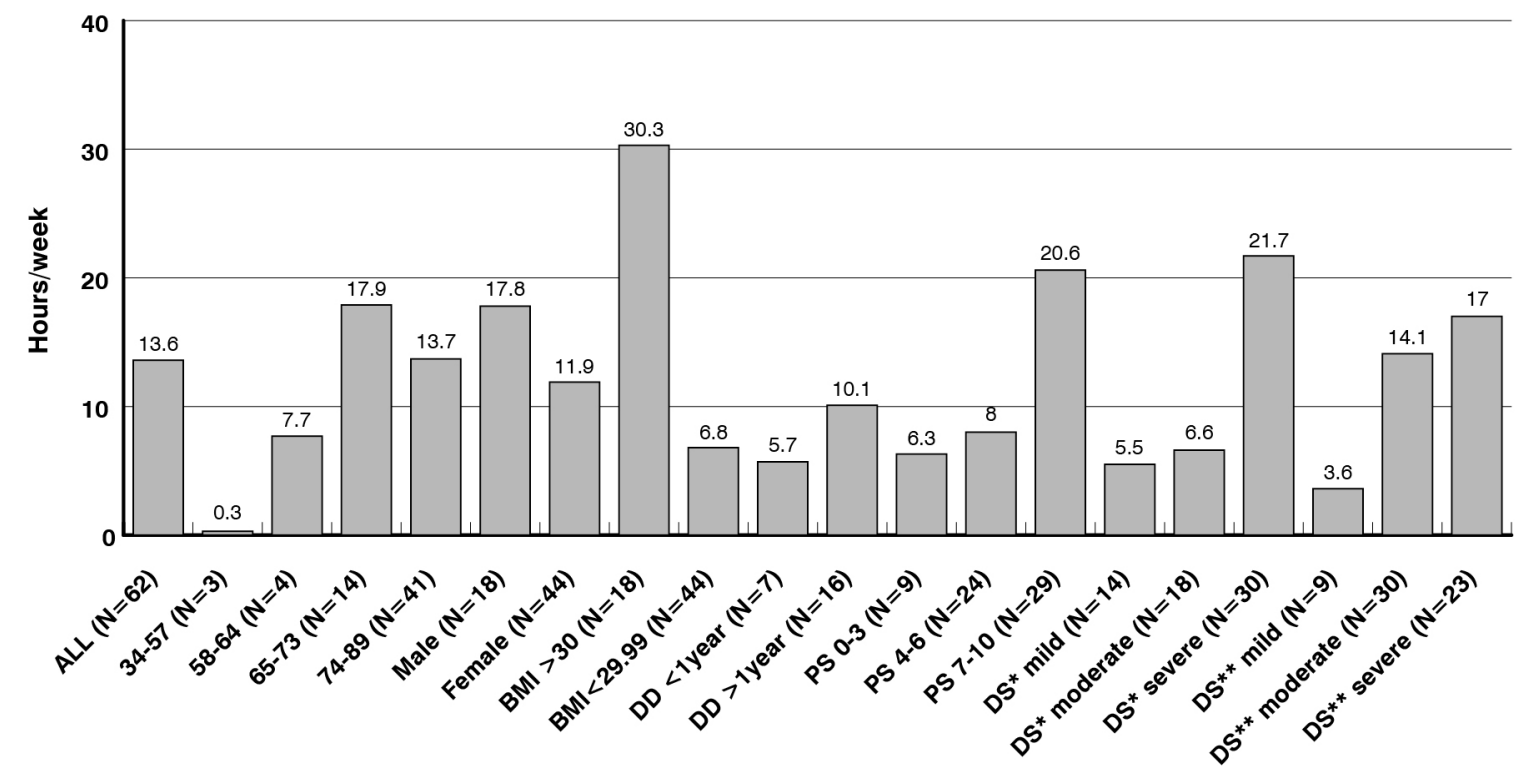

Figure 10. Hours family and professional caregiver

$\mathrm{BMI}=$ Body mass of index; DD = disease duration; DS* $=$ Disease Severity assessed by patient; DS** $=$ Disease Severity assessed by physician PS = Pain Severity

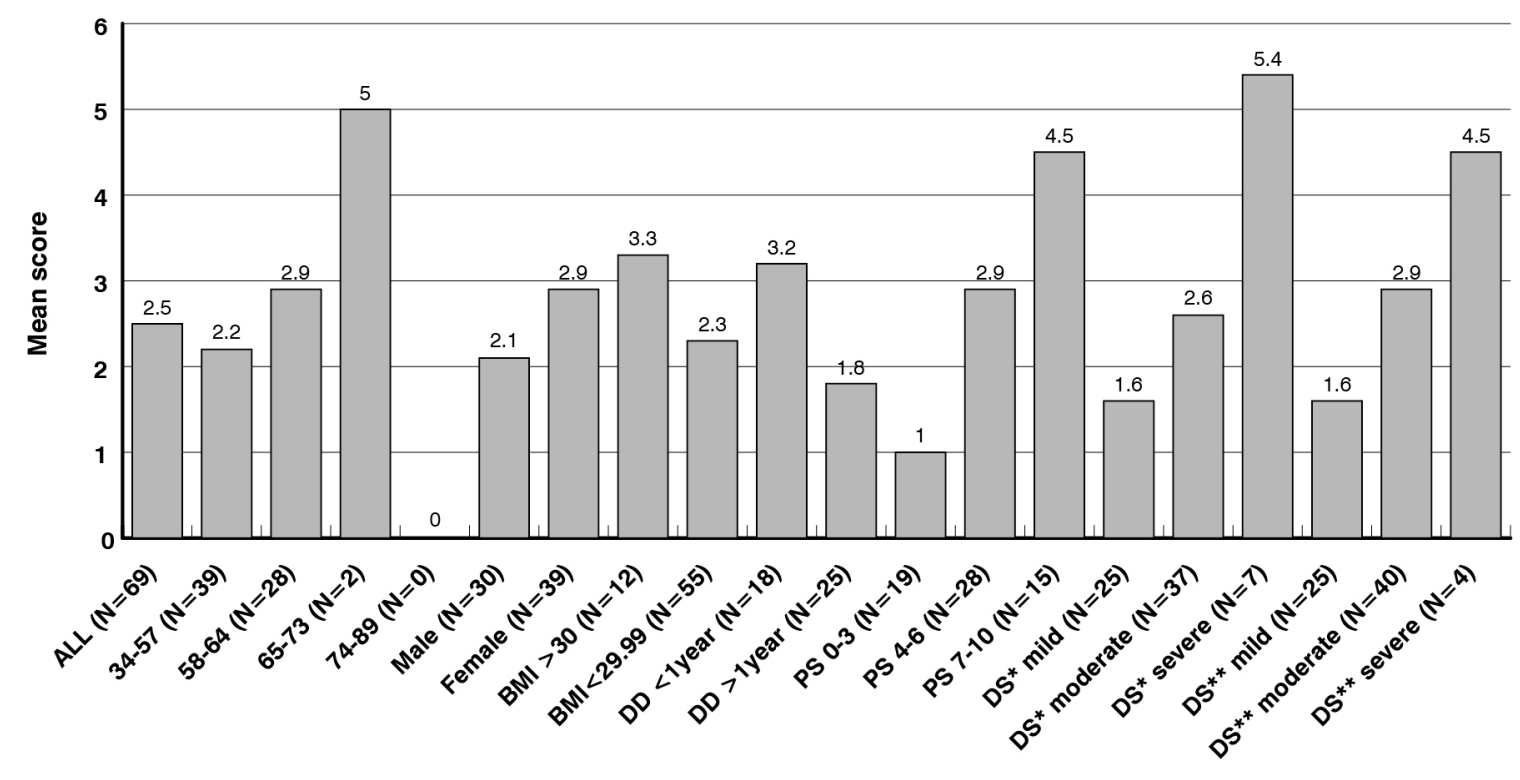

Figure 11. Work productivity

$\mathrm{BMI}=$ Body mass of index; DD = disease duration; DS* = Disease Severity assessed by patient; DS** = Disease Severity assessed by physician; PS = Pain Severity 


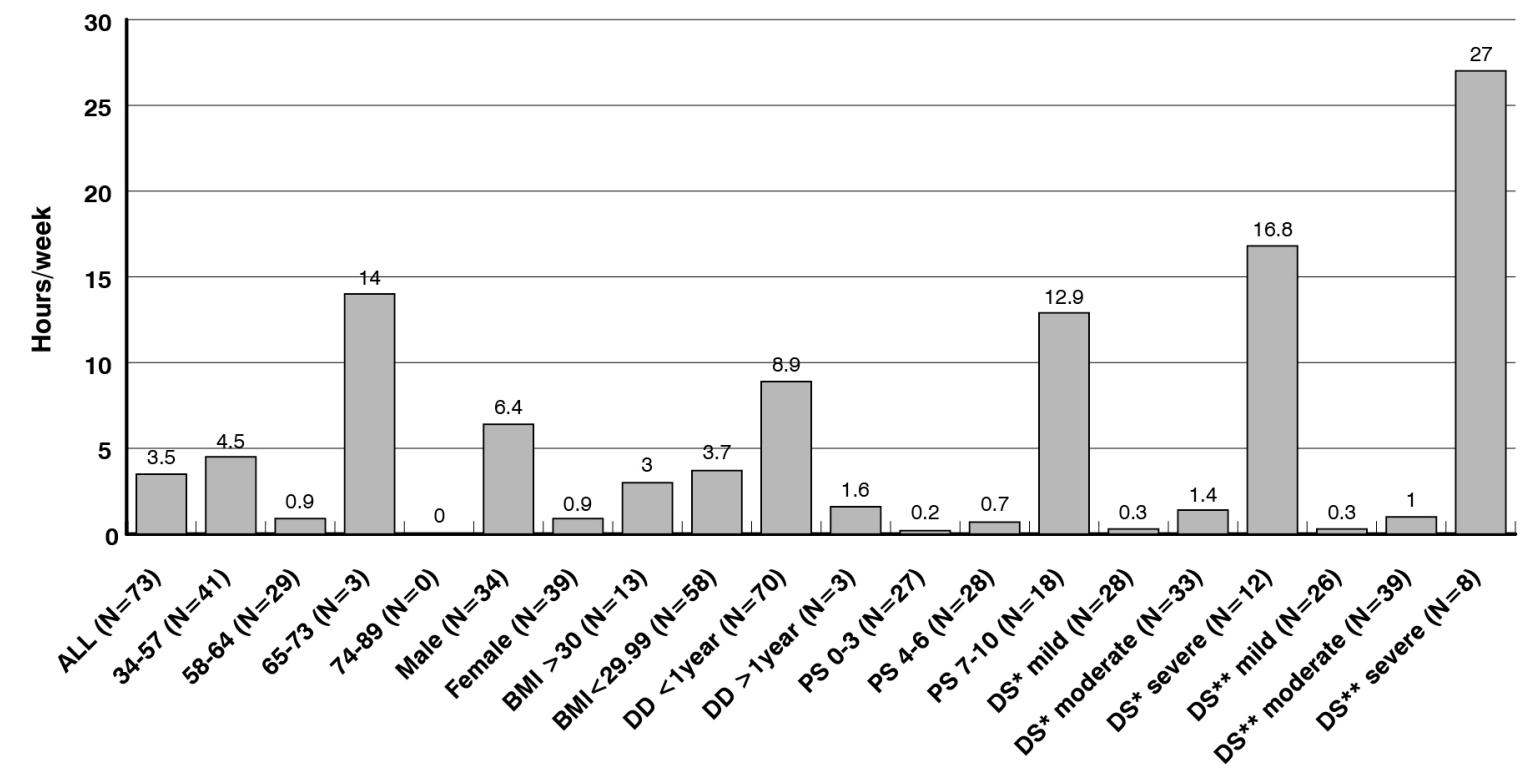

Figure 12. Hours missed from work

$\mathrm{BMI}=$ Body mass of index; DD = disease duration; $\mathrm{DS}^{\star}=$ Disease Severity assessed by patient; DS ${ }^{\star \star}=$ Disease Severity assessed by physician; PS = Pain Severity

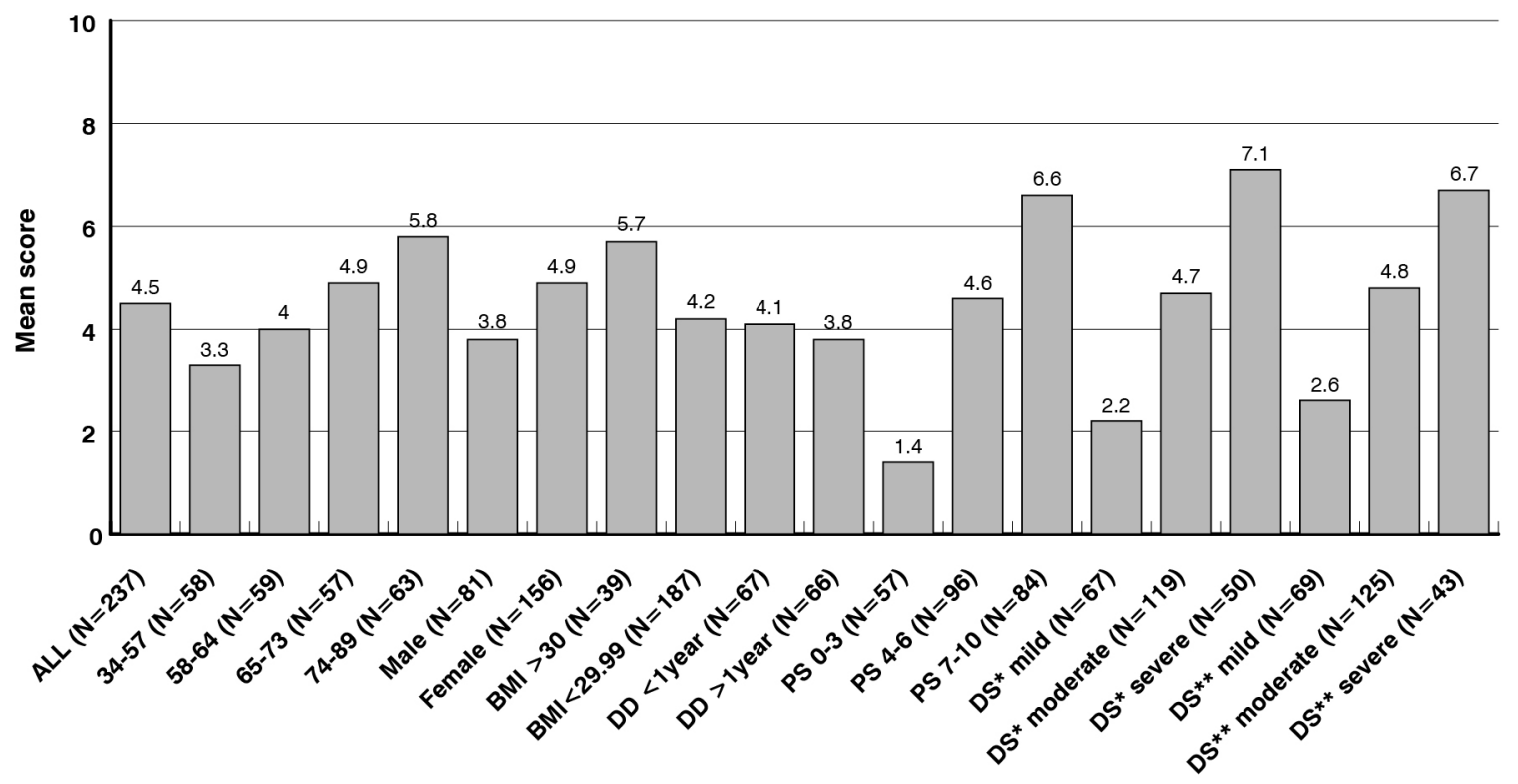

Figure 13. Ability to perform daily activities

$\mathrm{BMI}=$ Body mass of index; $\mathrm{DD}=$ disease duration; $\mathrm{DS}^{*}=$ Disease Severity assessed by patient; $\mathrm{DS}^{\star \star}=$ Disease Severity assessed by physician; PS = Pain Severity

All $(n=237)$ 